\title{
Cavity expansion-contraction based method for tunnel-soil-pile interaction in a unified clay and sand model: drained analysis
}

${ }^{1}$ Associate Research Scientist, State Key Laboratory for GeoMechanics and Deep Underground Engineering, School of Mechanics and Civil Engineering, China University of Mining and Technology, No.1 Daxue Road, Xuzhou, Jiangsu, 221116, China. ORCID number: 0000-00021469-4838. Email: pinqiang.mo@cumt.edu.cn

${ }^{2}$ Associate Professor, Faculty of Engineering, University of Nottingham, Nottingham NG7 2RD, UK. Email: Alec.Marshall@nottingham.ac.uk

${ }^{3}$ Professor, Key Laboratory of Transportation Tunnel Engineering (Southwest Jiaotong Univ.), Ministry of Education, Chengdu, 610031, China (corresponding author). Email: fy980220@swjtu.cn

\section{Abstract}

This paper proposes an analytical method based on drained solutions of cavity expansion and contraction in a unified clay and sand model to investigate tunnel-soil-pile interactions. Cavity expansion analyses are used to evaluate the effects of pile installation on ground stresses and to determine pile end bearing capacity and the distribution of shaft friction. Cavity contraction methods were adopted to replicate the tunnel convergence-confinement response using the singularity and image method for ground loss and ovalization of a shallow tunnel in a semiinfinite medium. A 2D model was developed which evaluates changes in mean stress and specific volume during pile installation and tunnel excavation. Outcomes from the developed analytical approach are compared against data from centrifuge tests in silica sand; results demonstrate that trends in pile load capacity degradation, mobilized safety factor, and tunneling induced pile settlement can be satisfactorily predicted for the case of a tunnel excavated beneath a pile with a constant service load. Criteria based on pile capacity, safety factor, and settlement 
are proposed which can be used to determine a critical tunnel volume loss or evaluate pile safety level. The paper contributes to the understanding of tunnel-soil-structure interaction mechanisms and provides an efficient means of conducting a preliminary risk assessment of tunnel-pile interaction.

Keywords: tunnel-soil-pile interaction, cavity expansion, pile capacity, safety factor, pile settlement

\section{Introduction}

Tunneling has an important role in urban construction to address the rapidly increasing demands and utilization of underground space, especially for transportation systems in congested urban areas (Mair, 2008; Kolymbas, 2008). Tunnelling induced ground movements are arguably inevitable given that excavations lead to stress release within the surrounding soil (Peck, 1969; Mair, 1979; Attewell et al., 1986; Gonzalez and Sagaseta, 2001; Marshall et al., 2012; Zhou, 2014; Mo and Yu, 2017b; Franza et al., 2019; Zhang et al., 2020). Both ground movements and stress release can have significant impacts on the serviceability and stability of surrounding structures (Burland et al., 1977). In urban areas, pile foundations often support superstructures, and it is common for new tunnel construction to occur in the proximity of existing piles. The tunnel-soil-pile interaction problem therefore becomes an issue of concern for engineers tasked with avoiding tunneling induced damages (Loganathan et al., 2000; Marshall and Mair, 2011; He et al., 2013; Basile, 2014).

Field trials in London clay have demonstrated that piles located directly above a tunnel experience much larger settlement than the ground, and the influence is largely dependent on the pile tip location in relation to the tunnel (Selemetas, 2005). Results of centrifuge tests have provided additional observations of the response of piles around new tunnels (Loganathan et al., 2000; Jacobsz, 2002; Williamson, 2014); a schematic of the influence zones around a new tunnel was provided by Jacobsz et al. (2004) based on tunneling induced pile settlement. The critical tunnel volume loss associated with pile failure was then evaluated according to the 
location of the pile tip within the influence zones. Further studies have reported that the tunnelsoil-pile interaction is also related to (1) soil type and drainage condition; (2) tunnel diameter and volume loss; and (3) pile length, roughness, and installation approach (Zhang et al., 2011; Dias and Bezuijen, 2015; Williamson et al., 2017; Franza and Marshall, 2017; Dias and Bezuijen, 2018; Franza and Marshall, 2018; Zhang et al., 2018, 2019).

Although numerical methods can simulate complex tunnel-soil-structure interaction problems (Mroueh and Shahrour, 2002; Zhang and Zhang, 2013; Jongpradist et al., 2013), their use in industry is constrained by issues related to model complexity, necessary computational time, and for tunneling-related problems, their ability to replicate greenfield settlements. Alternatively, analytical solutions have been developed for the estimation of tunneling induced ground movements and tunnel-soil-pile interactions, which are well suited to the simplified methods often used in practice, especially for preliminary design and risk assessment purposes (Loganathan and Poulos, 1998; Huang et al., 2009; Marshall, 2012). Conventional two-stage analytical approaches adopt a given input of greenfield soil movements to estimate pile responses using Winkler-based methods, and neglect the effect of stress relaxation and the interactions between the tunnel and pile (Basile, 2014; Franza et al. 2017). The cavity expansion method has been used to provide elasto-plastic analyses of tunnel-pile interactions by combining cavity expansion and contraction solutions in Mohr-Coulomb materials (Marshall, 2012, 2013; Marshall and Haji, 2015). However, the current analyses are only valid for perfectly plastic materials without consideration of void ratio changes, and the prediction of tunneling induced pile settlement is not provided.

To further develop the available tunnel-soil-pile interaction analyses based on cavity expansion methods, this paper presents an analytical method based on cavity expansion theory in association with critical state soil mechanics (Schofield and Wroth, 1968). A unified clay and sand model (Yu, 1998) is adopted in this study, which introduced two additional material constants into the standard Cam-clay models to simulate behaviour of both clay and sand. The drained cavity expansion and contraction solutions in the unified clay and sand model, developed by Mo and Yu (2018) and Yu et al. (2019), are employed to establish geometric and 
mechanical models for both pile installation and tunnel excavation. Pile stability is evaluated based on a non-linear load-settlement response, and tunneling induced ground settlement is determined based on solutions for shallow tunnels using a singularity and image method. As such, the effect of tunneling on pile capacity degradation, mobilized safety factor, and pile settlements are investigated. Analytical predictions are compared against available centrifuge test data for verification of the proposed method, and criteria for evaluation of critical tunnel volume loss are proposed. The proposed analytical approach provides a computationally inexpensive means of conducting preliminary risk assessments for the case of single piles with a constant service load that are affected by tunneling.

\section{Drained cavity expansion and contraction solutions in CASM}

Following the concepts of critical state soil mechanics, a unified state parameter model was proposed by Yu (1998) aiming to capture the overall behavior of both clay and sand under various drainage and loading conditions (referred to as CASM: clay and sand model). The standard Cam-clay model was extended with two additional material constants (i.e. spacing ratio $r^{*}$ and stress-state coefficient $n$ ) and reformulated in terms of the state parameter $\xi$, which serves as a key parameter for the behaviour of sands and over-consolidated clays (Been and Jefferies, 1985). Compared to the original Cam-clay model, the non-associated flow rule of CASM enables the prediction of peak deviatoric stress before critical state, the behavior for soils on the 'dry side' can be improved, and the softening and dilatancy of granular materials is captured. The extension to sandy soils also fits well to the drained analysis in this study for piling and tunneling problems in sands. Additionally, the standard Cam-clay models can be fully recovered, and the relative simplicity of CASM provides benefits in relation to further extension of the model and potential use within practical engineering applications. Note that the adopted CASM model cannot predict all features of soils, and further developments with additional model constants should be conducted to consider plastic deformations within the state boundary surface, anisotropy and destructuration of specific soils under various loading 
conditions.

110 Analytical solutions for drained expansion of both spherical and cylindrical cavities using

111 CASM were provided by Mo and $\mathrm{Yu}$ (2018). Correspondingly, the contraction solutions can be

112 readily obtained by modifying the expansion solutions with an unloading process, which is typically adopted for the analysis of underground excavations or tunnels in geomaterials (Yu et

114 al., 2019). The solutions were derived considering large deformations in the plastic stage together with logarithmic strain definitions, eventually providing distributions and evolutions

116 of stresses and strains around a cavity with an arbitrary expansion or contraction process. Fully

117 drained analysis is applied in this paper, neglecting the effect of pore water pressure, and compression positive notation is used throughout.

In this paper, results from a series of reference tests for both cavity expansion and contraction are provided to illustrate the stress paths and volumetric evolution of the surrounding soil. A

121 parameter $m$ is used to distinguish the spherical and cylindrical scenarios in this study, i.e. $m=1$ for a cylindrical cavity and $m=2$ for a spherical cavity. A set of reference constants are selected to model the behavior of Leighton Buzzard Fraction E sand based on $\mathrm{Hu}$ (2015), as follows: elastic constants $(\kappa=0.005, \mu=0.16)$; critical state constants $(M, \lambda=0.025$, $\Gamma=1.8)$; CASM constants $\left(r^{*}=33, n=2.0\right)$, where $M$ is determined from the constant-

126 volume friction angle of conventional triaxial tests $\phi_{t x}=32^{\circ}$ following Eq. (1).

$$
M=\frac{2(m+1) \sin \phi_{c s}}{(m+1)-\zeta \cdot(m-1) \sin \phi_{c s}}
$$

where

$$
\phi_{c s}=\left\{\begin{array}{ll}
\phi_{t x} & (\text { spherical }) \\
1.125 \phi_{t x} & (\text { cylindrical })
\end{array} \text { and } \zeta=\left\{\begin{aligned}
1 & \text { (expansion) } \\
-1 & \text { (contraction) }
\end{aligned}\right.\right.
$$

130 For determination of soil state, relative density is used to estimate the initial void ratio $e_{0}$, 131 according to $e_{\max }=1.014$ and $e_{\min }=0.613$ (after Franza, 2016). The initial state parameter is determined as $\xi_{0}=v_{0}+\lambda \ln p_{0}^{\prime}-\Gamma$, where $p_{0}^{\prime}$ is the initial mean stress condition. When the derived initial isotropic over-consolidation ratio $R_{0}=r^{*} / \exp \left[\xi_{0} /(\lambda-\right.$ $\kappa)]$ is less than 1 , the magnitude of initial specific volume $v_{0}=e_{0}+1$ is modified to keep 
the soil as normally consolidated.

136 Fig. 1 presents the results of cavity expansion and contraction analyses for both spherical and cylindrical scenarios in a soil with $R_{0}=1.1$ and $p_{0}^{\prime}=120 \mathrm{kPa}$ (i.e. $\xi_{0}=0.068$ and $v_{0}=$

138 1.744). Note that the calculation of expansion was formulated as a displacement control sequencing of the loading process with $a / a_{0}$ ( $a$ is the radius of cavity; subscript 0 indicates

140 the initial value) ranging from 1 to 20 , while the contraction process was programmed as a

141 pressure control sequencing of the unloading process, with cavity pressure $\sigma_{r, a}^{\prime}$ decreasing

142 from $p_{0}^{\prime}$ to $1 \mathrm{kPa}$ (the reference stress applied in critical state soil mechanics). The mean and 143 deviatoric stresses for symmetric cavity problems are defined as

$$
p^{\prime}=\frac{\sigma_{r}^{\prime}+m \cdot \sigma_{\theta}^{\prime}}{1+m} ; q=\sigma_{r}^{\prime}-\sigma_{\theta}^{\prime}
$$

where $\sigma_{r}^{\prime}$ and $\sigma_{\theta}^{\prime}$ are radial and tangential stresses, respectively. Fig. 1(a,b) show the stress paths in $q-p^{\prime}$ space for both spherical and cylindrical cavity expansion and contraction. The critical states are reached during the expansion process, and the stress state after unloading tends to approach the origin. Correspondingly, the evolution of specific volume is shown in $v-p^{\prime}$ space in Fig. 1(c,d). Cavity expansion leads to a decrease of void ratio (densification), whereas cavity contraction has little effect on specific volume. The normalized cavity pressure $\sigma_{r, a}^{\prime} / p_{0}^{\prime}$ versus $a / a_{0}$ data in Fig. 1(e,f) shows that the spherical case causes a larger change of cavity pressure for a given change in cavity size compared to the cylindrical case.

\section{Cavity expansion-contraction based method for tunnel-soil-pile}

\section{5 interaction}

\section{Geometric and mechanical models}

157 The tunnel-soil-pile interaction is simplified as a two-dimensional problem with a circular 158 tunnel under the vicinity of a single cylindrical pile, following the approach of Marshall and 159 Haji (2015), as shown in Fig. 2. The problem assumes that the single pile with radius $r_{p}$ is 
installed at depth to pile tip $z_{p}$, and a circular tunnel with radius $r_{t}$ is then excavated at a depth to its axis level $z_{t}$. The geometric distance between the tunnel center and pile tip is described by the horizontal distance $x_{t p}$ and vertical distance $z_{t p}=z_{t}-z_{p}$, thus distance $d_{t p}=$ $\sqrt{x_{t p}{ }^{2}+z_{t p}{ }^{2}}$. The $2 \mathrm{D}$ model in Fig. 2 is assumed as a critical scenario to consider the interactions between tunnel and pile within the $2 \mathrm{D}$ plane. However, a single pile is an axisymmetric (or quasi-axisymmetric) structure, and the tunnel is typically taken as a planestrain model ignoring the effects of excavation process. The tunnel-soil-pile interactions are therefore considered through the changes in mean stress and void ratio fields of the 2D plane to evaluate separately the influence of the processes of piling and tunneling, which is consistent with Marshall (2012), Marshall and Haji (2015) and Marshall et al. (2020). This hybrid 2D model assures a simple approach for analyzing the complex problem, thus the 3D effects associated with the responses of excavation process require further developments and validations to confirm the feasibility.

In this paper, the tunnel is located under the pile tip level to examine, in particular, the influence of tunneling on the degradation of pile load capacity and vertical settlement. The tunnel-soilpile interaction problem is analyzed using a mechanical model based on the combination of cavity expansion and contraction analyses. The interaction between the pile and tunnel is determined based on predicted changes to the surrounding soil (e.g. mean stress and void ratio) caused by both pile installation and tunnel volume loss. A calculation flow chart is provided in Fig. 3; reference to Stages and Steps in the subsequent text relate to Fig. 3. Correspondingly, a Matlab-based program is formulated following the calculation procedure of Fig. 3 to realize the analysis of the tunnel-soil-pile interaction problem. After setting the initial conditions (Stage 1), the pile is installed and changes to the mean stress and void ratio fields are calculated (Stage 2). Both installation resistance and pile load capacity are estimated using spherical cavity expansion in Stage 2. Tunnel excavation is simulated in Stage 3 by cylindrical cavity contraction, and the updated soil states are then used for the re-evaluation of pile capacity and settlement. Note that the concept of cavity expansion/contraction is embedded in the whole calculation process, which will be explained in detail in the following sections. 
189 Installation of displacement piles (i.e. driven or jacked) will cause significant changes to the stress profile in the surrounding soil. In Step 2.1 from Fig. 3, the installation resistance is

191 assumed to be equivalent to the cone tip resistance of a CPT (cone penetration test) at a certain depth (White and Bolton, 2005), and is estimated by spherical expansion of a cavity from an initial size comparable to that of the mean soil particle size to that of the size of the pile or probe. The cavity pressure $P_{a, s p h}$ approaches a critical state value for large expansion, which is used to estimate the cone tip resistance $q_{c}$ following Ladanyi and Johnson (1974) and Suzuki and Lehane (2015).

$$
q_{c}=P_{a, s p h} \cdot\left(1+\sqrt{3} \tan \phi_{t x}\right)
$$

Pile shaft friction $\tau_{s}$ is then determined in Step 2.2 using the CPT-based design method UWA05 for driven closed-ended piles in siliceous sand (Lehane et al., 2005):

$$
\sigma_{r, s}^{\prime}(z)=0.03 \cdot q_{c} \cdot\left[\max \left(\frac{z_{p}-z}{b_{p}}, 2\right)^{-0.5}+\Delta \sigma_{r d}^{\prime}\right]
$$

$$
\tau_{s}(z)=\sigma_{r, s}^{\prime}(z) \cdot \tan \delta_{f}
$$

where $\sigma_{r, s}^{\prime}$ is the normal stress along the shaft surface, $b_{p}$ is pile diameter, and

$$
\Delta \sigma_{r d}^{\prime}=2 G \cdot \Delta y / r_{p}, \Delta y \sim 0.02 \mathrm{~mm}, \delta_{f} \sim \phi_{t x}-5^{\circ}
$$

It is worth noting that $\Delta \sigma_{r d}^{\prime}$ is calculated based on elastic cylindrical cavity expansion, and $\Delta y$ is used to consider the dilatant expansion of the shear band around pile shaft. $\delta_{f}$ is the interface friction angle after Randolph et al. (1994), assumed as $\phi_{t x}-5^{\circ}$. According to Mo and $\mathrm{Yu}(2017 \mathrm{a})$, the shear modulus is defined as follows, which varies with the local stress

208 condition and specific volume:

$$
G=\frac{(1+m)(1-2 \mu) v p \prime}{2[1+(m-1) \mu] \kappa}
$$

where $\mu$ is the Poisson's ratio.

For predictions of mean stress and void ratio fields in Step 2.3, the soil is separated into two 
regions (i.e. below pile tip and above pile tip), and their changes from each step are simply combined to yield the cumulative fields. Spherical cavity expansion at the pile tip is used for predictions below the pile tip, whereas cylindrical cavity expansion along the pile shaft is used above the pile tip. Details of the calculations in each region are provided in the following.

As the initial states around the pile tip (i.e. $p_{0, t i p}^{\prime}$ and $v_{0, t i p}$ ) are adopted for the spherical cavity expansion calculation with the assumption of isotropic conditions, the changes of mean stress and specific volume in the surrounding soil below the pile tip require modification based on a simple proportional criterion to their in-situ states:

$$
\Delta p^{\prime}=\frac{\Delta p_{s p h}^{\prime}}{p_{0, t i p}^{\prime}} \cdot p_{0}^{\prime} ; \Delta v=\frac{\Delta v_{s p h}}{v_{0, t i p}} \cdot v_{0}
$$

where $\Delta p_{s p h}^{\prime}$ and $\Delta v_{s p h}$ are obtained from the spherical cavity expansion results according to the distance to pile tip, and $p_{0}^{\prime}$ and $v_{0}$ are the current states of mean stress and specific volume of a soil element. Note that the in-situ states are only used for the steps related to pile installation (Steps 2.1 and 2.2); updated stress and specific volume fields are used for estimation of pile capacity (Step 2.4) and during tunneling (Stage 3).

The distribution of mean stress above the pile tip after pile installation is estimated using the obtained normal stress on the pile shaft $\sigma_{r, s}^{\prime}$ (Eq. 4a), following the pattern obtained from elastic cylindrical cavity expansion (i.e. $\left.\sigma_{r}^{\prime}=p_{0}^{\prime}+\left(\sigma_{r, S}^{\prime}-p_{0}^{\prime}\right) \cdot\left(r_{p} / r\right)^{2}\right)$. The void ratio distribution within soil horizons up to the pile tip depth are set to be identical to that at the pile tip depth (for example, see Fig. 4b). For non-displacement (bored) piles, stress and void ratio around the pile are assumed to remain unchanged during pile installation, and the shaft friction is calculated as $\tau_{s}=\sigma_{v 0}^{\prime} \cdot K_{0} \cdot \tan \delta_{f}$ ( $K_{0}$ is the at-rest lateral earth pressure coefficient).

In Step 2.4, pile end bearing capacity is predicted by spherical expansion of a cavity with initial radius $r_{p}$. The magnitude of expansion was set to $10 \%$ (i.e. to $1.1 r_{p}$ ), relating to the determination of pile capacity for a settlement equivalent to $10 \%$ of the pile diameter (Lehane et al., 2005; White and Bolton, 2005). The correlation between pile end bearing capacity and spherical cavity pressure from Vesic (1977) and Randolph et al. (1994) was adopted in this 
study.

$$
q_{t}=P_{a, s p h} \cdot\left(1+\tan \alpha \tan \phi_{t x}\right) \quad \text { where } \alpha=45^{\circ}+\phi_{t x} / 2
$$

240 Pile end bearing capacity is strongly dependent on the soil stress field, which is modified by 241 the actions of both pile installation and tunnel volume loss. To account for pile installation 242 effects, the initial stress condition for the spherical cavity expansion from $r_{p}$ to $1.1 r_{p}$ in Step 2432.4 was assumed as the average value within the plastic zone of soil around the pile tip from 244 the pile installation spherical cavity expansion analysis (i.e. Step 2.1). Pile load capacity is then obtained as:

$$
Q=Q_{\text {tip }}+Q_{\text {shaft }}=q_{t} \cdot \pi \cdot r_{p}^{2}+\int_{0}^{z_{p}} \tau_{s} d z \cdot \pi \cdot b_{p} \quad \text { where } b_{p}=2 r_{p}
$$

\section{Tunneling and tunnel-soil-pile interaction}

Cylindrical cavity contraction is used to simulate the process of tunneling, providing the convergence-confinement curve (Step 3.1). The effect of the tunnel face can be analyzed by spherical cavity contraction; however this paper focuses on a two dimensional model taking the tunnel as a cylindrical tube. Note that the initial stress and specific volume are assumed as the average value from the updated stress and specific volume fields from Stage 2 within the range of $5 r_{t}$ from the tunnel center. The cavity contraction solution provides information on soil response in terms of both stress state and tunneling induced ground deformations. Regarding a shallow tunnel in a semi-infinite medium, the conventional concentric displacement field around a cavity does not provide an accurate representation of real ground displacements around shallow tunnels (Logonathan and Poulos, 1998). To account for this, an elastic solution for compressible material based on a singularity and image method, generalized from Sagaseta (1987) and proposed by Verruijt and Booker (1996), was adopted for the calculation of displacements. Both ground loss and ovalization of the tunnel are considered in the solution, and the modification of Strack (2002) was applied in this study to remove the tangential displacements at the cavity boundary. Therefore, for a certain tunnel volume loss $V_{l, t}$, the displacement field provided by the elastic solution is employed to evaluate the relative soil movements, which are then used to calculate the changes of mean stress and void ratio based 
on elastic-plastic cavity contraction analysis (Step 3.2; an example demonstrating this process is provided in the next section). As was done for the pile installation stage, a stress reduction ratio is applied to the current stress field (after pile installation), and specific volume is changed accordingly:

$$
\Delta p^{\prime}=\frac{\Delta p_{c y l}^{\prime}}{p_{0, t u n}^{\prime}} \cdot p_{0}^{\prime} ; \Delta v=\frac{\Delta v_{c y l}}{v_{0, t u n}} \cdot v_{0}
$$

With the updated stress and void ratio fields, the pile end bearing capacity is re-evaluated in Step 3.3. Again assuming an initial state based on the average values within the plastic region around the pile tip during the installation process, the post-tunneling pile end bearing capacity $q_{t, V l}$ is determined from spherical cavity expansion. For the estimation of shaft friction of displacement piles after tunneling, the reduced cone tip resistance is assumed to be equivalent to the reduced pile end bearing capacity (i.e. $q_{c, V l} \sim q_{t, V l}$ ). Additionally, tunnel volume loss leads to the reduction of stresses and increase of void ratio around the pile shaft, as well as the reduction of soil shear stiffness. The reduced pile load capacity is referred to as $Q_{V l}=Q_{t i p, V l}+$ $Q_{\text {shaft,Vl}}$. To represent the pile load capacity degradation, the reduction factor for total capacity is defined as:

$$
R_{Q}=Q_{V l} / Q_{0}
$$

Underground excavation induces ground movements, and the stress relaxation in the surrounding soil can reduce the capacity of adjacent piles. Therefore, tunneling induced pile settlement $s_{p i l e, V l}$ in Step 3.4 is considered as a combination of two components: (1) tunnel volume loss induced ground settlement, $s_{1, V l}$, and (2) pile capacity degradation induced pile settlement, $s_{2, V l}$. Tunneling induced ground settlement $s_{1, V l}$ is estimated from the vertical component of the ground displacement field at the pile tip based on the analytical solution for shallow tunnels using the singularity and image method (Strack, 2002). For the prediction of post-tunneling soil strength-loss induced settlement $s_{2, V l}$, the non-linear pile load-settlement response was assumed as a hyperbolic asymptote curve, following Chin (1971):

$$
q=\frac{s / b_{p}}{1 / k_{i}+s / b_{p} / q_{t}} \quad \text { where } k_{i}=\frac{8 G}{\pi(1-\mu)}
$$


291 Note that $G$ is estimated from the updated stress field caused by the variation of tunnel volume 292 loss using Eq. (5). Therefore, with a constant pile service load ( $P_{\text {load }}=Q_{0} / S F_{0}, S F_{0}$ is the 293 initial safety factor), the initial settlement is determined as:

$$
s_{0}=\frac{q_{t} / k_{i}}{q_{t} / q_{s e r v, 0}-1} \cdot b_{p} \quad \text { where } q_{s e r v, 0}=\frac{\max \left(P_{\text {load }}-Q_{\text {shaft }}, 0\right)}{\pi \cdot r_{p}^{2}}
$$

295

After tunnel volume loss, the safety factor is reduced to $S F_{V l}=Q_{V l} / P_{l o a d}$, and the posttunnelling settlement is calculated as:

$$
s_{i}=\frac{q_{t, V l} / k_{i, V l}}{q_{t, V l} / q_{s e r v, V l}-1} \cdot b_{p} \quad \text { where } q_{s e r v, V l}=\frac{\max \left(P_{\text {load }}-Q_{\text {shaft }, V l}, 0\right)}{\pi \cdot r_{p}^{2}}
$$

Post-tunneling pile strength induced settlement is estimated as $s_{2, V l}=s_{i}-s_{0}$, and the total post-tunneling pile settlement is calculated as:

$$
s_{\text {pile }, V l}=s_{1, V l}+s_{2, V l}
$$

Note that the work presented in this paper focuses on tunnel interaction with single piles with a constant load applied to the pile. The analysis does not consider pile interactions within a group or load redistribution due to a connected superstructure, which can have an impact on the tunnel-pile interactions (Franza and Marshall, 2019).

\section{Comparison with centrifuge test data}

Results from centrifuge tests of tunneling under a single pile are used for a verification exercise of the proposed analytical solutions for tunnel-soil-pile interaction. Three independent test series in dry silica sand with different relative densities were conducted by Jacobsz (2002), Marshall (2009), and Franza (2016), using a similar technique for the simulation of tunnel volume loss. Centrifuge test results are presented here in model scale unless otherwise stated. Leighton Buzzard Fraction E sand with an average particle size of $d_{50}=0.122 \mathrm{~mm}$ was used in all centrifuge tests. The soil model parameters presented in the previous section were used for the sand. The unit weight of the soil was determined based on the initial void ratio, and the initial mean stress condition at depth $z$ was $p_{0,0}^{\prime}=\gamma \cdot z \cdot N_{g} \cdot\left(1+2 K_{0}\right) / 3$, where $N_{g}$ is the 
centrifuge scaling factor and $K_{0} \approx 0.5$. Results from dense sand tests are presented first to illustrate the calculation process and to compare with results from Marshall (2009). Comparisons with loose sand tests (Franza, 2016) and medium dense sand tests (Jacobsz, 2002) are then provided. Note that only results for displacement (driven) piles are presented here, and a constant load was applied to each single pile, with initial safety factor evaluated based on the measured resistance during the driving of the pile.

\section{Dense sand tests}

Dense sand tests with relative density $D_{R} \approx 90 \%$ were carried out by Marshall (2009); the centrifuge model properties are shown in Table 1. After replicating the initial stress field within the centrifuge model, the initial void ratio was kept as $e_{0}=0.653$, and the initial specific volume field was generated by the relation $v_{0}=e_{0}+1$ (Step 1.3). Note that the initial state parameter $\xi_{0}$ increases with depth.

Test TP1-P1, in which the tunnel is located directly below the pile (i.e. $x_{t p}=0$ ), is taken as an example to illustrate the calculation steps following the proposed method in the previous section and Fig. 3. Additionally, a worked example for TP1-P1 is provided in the "Supplementary Materials" to provide details in the step-by-step calculation. To consider the pilling induced changes in the stress field following the steps in the subsection of "Pile installation and load capacity”, Fig. 4a shows the mean stress field after pile installation (Step 2.3). The stress concentration is mostly located in the vicinity of the pile, especially around the pile tip, which is consistent with experimental observations of piling induced soil deformation (White and Bolton, 2004; Marshall and Mair, 2011). Stress levels around the pile shaft are also increased by installation, but with a much lower magnitude, which relates well to the effect of stress reduction at the pile shoulder (White and Bolton, 2004). The plastic region of spherical cavity expansion for pile installation is about $40 \mathrm{~mm}$, which is smaller than the distance to the tunnel lining $d_{l p}=d_{t p}-r_{t}=55 \mathrm{~mm}$, and the influence of pile installation at the location of the tunnel is therefore limited. On the other hand, the void ratio of the surrounding soil is decreased due to piling induced densification of the soil, as shown with the change of specific volume field in Fig. 4b. For this displacement pile, the penetration resistance at the pile tip is 
predicted to be $q_{c}=4.87 \mathrm{MPa}$ (Step 2.1), while the pile tip load capacity is $599 \mathrm{~N}$ and the pile-shaft capacity is about $780 \mathrm{~N}$ (Step 2.4). The total estimated pile capacity is therefore

$3461379 \mathrm{~N}$, compared to the experimental measurement of $1790 \mathrm{~N}$ (Marshall et al., 2020).

347 In Stage 3 of the method, tunnel excavation is simulated by cylindrical cavity contraction, and 348 in this case cavity pressure was decreased from its initial stress condition $p_{0, t u n}^{\prime}=151.9 \mathrm{kPa}$.

349 The equivalent tunnel volume loss $V_{l, t}$ can be determined as $\left[1-\left(a / a_{0}\right)^{2}\right] \times 100 \%$. The 350 cavity contraction response is shown in Fig. 5, which is typically referred to as a confinement351 convergence curve (Step 3.1), where the tunnel pressure $\sigma_{r, a}^{\prime}$ is normalised by the initial 352 pressure within the tunnel (in the centrifuge experiments, this was set to be the vertical stress 353 at the depth of the tunnel axis). The predicted ultimate value $V_{l, u l t}=1.76 \%$ indicates tunnel 354 convergence without support. The predicted cavity pressure is shown to reduce faster than what 355 was observed in the experiment. This may be related to the adequacy of the applied critical state 356 model, or that soil arching phenomena caused by tunneling (Franza and Marshall, 2019) are not 357 well replicated. Fig. 6 shows the change of mean stress and specific volume with respect to 358 normalized soil movement $u_{r} / r$. The mean stress decreases and specific volume increases with 359 tunnel excavation after a soil movement of about $u_{r} / r=0.0015$, which indicates the 360 initiation of the plastic stage in the soil around the excavation.

361 Although the cavity contraction in CASM has shown its ability to predict soil behavior around tunnels by Mo and $\mathrm{Yu}$ (2017b), the effects of the free ground surface for shallow tunnels were 363 neglected, and modifications are required to account for the uniform convergence, ovalization 364 and vertical translation (Gonzalez and Sagaseta, 2001). In terms of the cavity contraction in a 365 half-infinite space to include the surface effects, the closed-form solutions are limited to the 366 linearly elastic materials, following Verruijt and Booker (1996). Therefore, as a compromised 367 approach, the tunnel excavation problem is taken as a displacement-controlled process, and the 368 displacement field at the ultimate tunnel volume loss is obtained based on the elastic solution 369 in a semi-infinite medium (Strack, 2002). The displacement-induced changes are then predicted 370 from the elasto-plastic cavity contraction response according to the magnitude of deformation. 371 Note that this approach contains some arbitrary assumptions that may cause undesirable errors, 
and further adjustments can be applied to improve the predictions for shallow tunnels. As shown in Fig. 7, both vertical displacement and normalized displacement $\left(u_{r} / r\right)$ contours indicate that the soil above the tunnel experiences the largest deformation. The pattern above the tunnel in Fig. 7a is similar to that of centrifuge tests of greenfield tunneling (e.g. Marshall, 2009; Marshall et al. 2012; Franza et al., 2019). The normalized displacement in Fig. 7b is used to estimate the change of mean stress and void ratio of the surrounding soil based on the curves in Fig. 6, which represent the soil response due to cavity contraction. The normalized soil displacement at the pile tip is $u_{r} / r=0.0035$, which is larger than the critical value of $u_{r} / r=$ 0.0015 from Fig. 5, indicating that the soil at the pile tip is in a plastic state at the specified magnitude of tunnel volume loss. Based on the critical value of $u_{r} / r=0.0015$ for this scenario, Fig. $7 \mathrm{~b}$ also indicates that over half of the pile is located in the plastic region when the tunnel is fully unloaded $\left(V_{l, u l t}=1.76 \%\right)$. The mean stress and change in specific volume at this stage of tunnel volume loss are presented in Fig. 8. Compared to the stress field after pile installation (Fig. 4a), the stress reduction in Fig. 8a at $V_{l, u l t}$ is significant, however a small area with relatively high stresses still exists close to the pile tip compared to the in-situ stress field. The change of specific volume (Fig. $8 \mathrm{~b}$ ) shows that the soil becomes looser due to tunnel excavation, however the area around the pile tip retains a negative change in specific volume (soil densification) from the pile installation stage.

For a given magnitude of tunnel volume loss, the mean stress and specific volume fields are predicted in Step 3.2 and the shear stiffness is updated using Eq. (6). The pile end bearing capacity $q_{t, V l}$ is re-calculated based on Eq. (7) in Step 3.3, and the foundation stiffness $k_{i, V l}$ is obtained by updating the shear stiffness from Eq. (11). The degradation of the pile loadsettlement curve is shown in Fig. 9a following Step 3.4. With an increase of tunnel volume loss, both $q_{t, V l}$ and $k_{i, V l}$ decrease, and the updated load-settlement curve represents a virtual pile loading test which can be used for the estimation of pile settlement $s_{2, V l}$. Before tunneling, the pile shaft capacity is $Q_{\text {shaft }, 0}=780 \mathrm{~N}$ and the pile tip load capacity is $599 \mathrm{~N}$, hence to get the required initial safety factor $S F_{0}=1.65$ to match the TP1-P1 centrifuge test (see Table 1), a service load at the pile head of $835.6 \mathrm{~N}$ is required. Shaft capacity is mobilized at much 
smaller displacements than end bearing capacity, hence it was assumed that only $Q_{0}-$ $Q_{\text {shaft }, 0}=835.6-780=55.6 \mathrm{~N}$ of load was carried by the pile tip (tip stress of about $490 \mathrm{kPa}$ ) prior to tunneling. This implies an initial normalized settlement $s_{0} / b_{p}=0.0035$ based on Eq. (12), which is negligible (see $q-s$ curve before tunnelling shown in Fig. 9a). When the tunnel volume loss reaches $1.0 \%$, for example, the pile tip stress increases to about $2500 \mathrm{kPa}\left(q_{\text {serv }, V l}=\max \left(P_{\text {load }}-Q_{\text {shaft }, V l}, 0\right) /\left(\pi \cdot r_{p}{ }^{2}\right)\right)$ according to Eq. (13), and the normalized settlement is $s_{i} / b_{p}=0.0659$ from the corresponding pile load-settlement curve (i.e. dark blue line in Fig. 9a). The pile capacity degradation induced pile settlement at $V_{l, t}=$ $1.0 \%$ is thus calculated as $s_{2, V l}=s_{i}-s_{0} \approx 0.75 \mathrm{~mm}$ (equivalent to $6.25 \%$ pile diameter and $56 \mathrm{~mm}$ in prototype scale). Together with the tunneling induced ground settlement at the pile tip (Fig. 7a), the total tunneling induced pile settlement is $s_{p i l e, V l}=s_{1, V l}+s_{2, V l} \approx 1.04 \mathrm{~mm}$ ( $8.7 \%$ pile diameter; $78 \mathrm{~mm}$ at porotype scale). A further increase of tunnel volume loss significantly accelerates the pile movements due to the degradation of the load-settlement curve and the increase of pile tip stress. The decrease of pile load capacity components (tip, shaft, and total) with tunnel volume loss are provided in Fig. 9b, showing that the trend of degradation of the pile tip and shaft are very similar, with a reduction of $59.6 \%$ and $65.1 \%$, respectively, at ultimate tunnel volume loss $V_{l, u l t}=1.76 \%$.

The predicted ground and pile settlements with tunnel volume loss are presented in Fig. 10a-b, with comparisons to centrifuge data from Marshall (2009). Generally, the predicted greenfield soil displacements are comparable with the experimental data, and the predicted tunneling induced pile settlement agrees well with experimental data, though pile failure in the experiment was more brittle than predicted. The variation of the reduction factor for total pile capacity $R_{Q}$ with $V_{l, t}$ is provided in Fig. 10c.

As a recommendation for critical tunnel volume loss, Marshall (2012) and Marshall and Haji (2015) proposed that a value of $R_{Q}^{f}=0.85$ could be used (based on the experimental data that was considered). Based on this somewhat empirical pile capacity criterion, a critical tunnel volume loss is obtained for test TP1-P1: $V_{l}^{f, R Q}=0.80 \%$ (as shown in Fig. 10c). This criterion 
is relatively conservative, as also indicated by Marshall et al. (2020), and the predicted normalized pile settlement is less than 0.03 for TP1-P1.

Considering the initial pile safety factor, a criterion for critical tunnel volume loss based on the post-tunneling safety factor was suggested by Franza and Marshall (2018) and Marshall et al.

431 (2020). This criterion requires a critical post-tunneling safety factor $S F^{f}$, where a value of 1.0 432 indicates the initiation of pile failure. For test TP1-P1, the critical tunnel volume loss based on 433 the safety factor criterion $V_{l}^{f, S F}$ is determined from the $R_{Q}-V_{l, t}$ curve when $R_{Q, S F}=$ $S F^{f} / S F_{0}=1 / S F_{0}=0.61$. The value of $V_{l}^{f, S F}$ for TP1-P1 is $1.2 \%$ (see Fig. 10c).

The above criteria are applied only when the degradation of pile capacity with tunnel volume loss is obtained. The mobilized pile capacity is difficult to measure in experimental or field tests, whereas a more direct measurement is related to tunneling induced pile settlements. A pile displacement or serviceability criterion can also be used based on the tunneling induced pile settlement. A critical normalized pile settlement of $s^{f} / b_{p}=0.1$ is used here, which

440 follows from the criterion of pile loading tests for determination of pile capacity. This critical settlement may relate to overly large displacements for cases with stringent serviceability limit state criteria (e.g. $s^{f}<20 \mathrm{~mm}$ as suggested by Jacobsz et al., 2004), however the value can be modified within the suggested approach depending on the application. The obtained critical tunnel volume loss based on the stated pile settlement criterion $V_{l}^{f, s}$ is $1.04 \%$ for test TP1-P1. Experimentally, pile failure was deemed to occur at the location of a distinct increase in magnitude of slope or curvature of the pile settlement versus tunnel volume loss curve, giving an experimental critical tunnel volume loss of $V_{l}^{f, \exp }=0.92 \%$, based on Marshall et al. (2020).

448 The resulting safety factor at $V_{l}^{f, s}$ is around 1.15 , illustrating how the analytical approach can 449 provide information of pile stability when applying the criterion based on pile settlements. Note 450 that this outcome is strictly related to the assumed input of greenfield settlement profile and 451 should not be generalized. In terms of the empirical parameters in the criteria for evaluating the stability of piles, a 
sensitivity study for TP1-P1 is presented in Fig. 11 to examine their viability. The variations of $R_{Q}, S F$ and $s / b_{p}$ against the tunnel volume loss are jointly shown in Fig. 11a, where the influence of empirical parameters for critical criteria can be found from the curves. It is noted that the critical reduction factor for total pile capacity $R_{Q}=0.85$ tends to yields conservative results of corresponding pile safety factor and settlement, and pile settlements are extremely large when the safety factor reduces to 1 . The pile settlement criterion with $s / b_{p}=0.1$ provides a balanced estimation of critical situation with $S F \approx 1.2$ and $R_{Q} \approx 0.6$. Fig. $11 \mathrm{~b}$ shows the linear correlation between $R_{Q}$ and $S F$, as well as the variation of $s / b_{p}$ against $R_{Q}$. The three indices indicate the tunneling induced influences in terms of pile capacity, safety factor and pile settlement, and they should be integrated to conduct evaluations for design. For practical use in a particular case, the empirical parameters in the criteria can be further adjusted to provide a comprehensive assessment of the tunnel-pile interaction.

The influence of tunnel-pile location on the mean stress and specific volume results is illustrated in Fig. 12 using the three dense sand centrifuge test scenarios from Table 1. Fig. 13 shows predictions of tunneling induced pile settlements and pile capacity reduction factors for the same tests, and compares results with the available experimental data. It can be seen that the contours of $\Delta p^{\prime}$ and $\Delta v$ are heavily dependent on the tunnel-pile location. When the pile is installed within the influence zone of tunneling for tests TP1-P1 and TP2-P1, the pile stability is significantly affected by the tunnel, as indicated in Fig. 13. The predicted pile settlement for TP2-P1 in Fig. 13a does not match the experimental data well after about 1\% tunnel volume loss, however the trend is sensible an is this case the prediction is conservative. Applying the empirical pile capacity criterion for test TP2-P1 (i.e. $R_{Q}^{f}=0.85$ ), the critical tunnel volume $\operatorname{loss} V_{l}^{f, R Q}$ is $1.51 \%$, as indicated in Fig. 13b. In comparison, the experimental data shows $V_{l}^{f, \exp }=2.4 \%$ for TP2-P1. The analysis also indicates that the tunnel converges at a tunnel volume loss of approximately $1.75 \%$, at which the predicted normalized pile settlement is less than the required value of 0.1 to apply the pile settlement criterion. However, based on the expected degradation trends (shown as extended portions of the predicted lines in Fig. 13a and 
b), estimations of $V_{l}^{f, S F} \approx 2.13 \%$ and $V_{l}^{f, s} \approx 1.96 \%$ for TP2-P1 were obtained. The pile in

481 TP1-P2 is not affected by tunneling because of its relative distance from the tunnel, which is demonstrated in the experimental and analytical results. When the pile is located beyond the plastic region of soil caused by tunneling, pile settlements are due solely to the tunneling induced ground movement, $s_{1, V l}$.

The above comparison with centrifuge data of dense sand tests provides a reasonable validation of the proposed cavity expansion-contraction based method for tunnel-soil-pile interaction. The analytical approach provides a rational method for predicting critical tunnel volume loss based on either a pile capacity or serviceability criterion. Again, it is important to note that the outcomes of the serviceability criterion are directly related to the assumed input of greenfield settlements, hence the results here should only be generally applied with appropriate judgement, or specific analyses using the described approach should be conducted.

\section{Loose sand and medium dense sand tests}

Further verification of the proposed method is presented in this section with comparisons to loose and medium dense sand centrifuge tests. Franza (2016) conducted the loose sand tests with $D_{R} \approx 30 \%$ (i.e. $e_{0}=0.8937$ ); the model parameters are listed in Table 2 with variations of horizontal offset $x_{t p}$ and initial safety factor $S F_{0}$. The Test ID is according to the pile position and $S F_{0}$, e.g. 'P1SF1.5' represents a test with a pile installed at Position $1\left(x_{t p}=\right.$ $0 \mathrm{~mm})$ and loaded with $S F_{0}=1.5$.

The predicted results for the loose sand tests are presented in Fig. 14, along with the centrifuge test data from Franza (2016). Fig. 13a-c show the greenfield ground settlements at the positions of the pile head (i.e. ground surface) and pile tip. The decrease of ground settlement with horizontal offset is predicted reasonably well, however the analytical input of the variation of greenfield settlement with depth could be improved. The settlement at the pile tip has the larger influence on predictions, and it shows relatively good agreement with experiments.

The tunneling induced pile settlements for piles with $S F_{0}=1.5$ and 2.5 are illustrated in Fig. $14 d-f$, showing that the initial safety factor plays an important role during tunnel excavation. 
For tests with $x_{t p}<75 \mathrm{~mm}$, piles with higher $S F_{0}$ show signs of failure at a larger tunnel volume loss than those with lower $S F_{0}$, in agreement with the experimental results. Although the comparisons give similar trends of tunneling induced pile settlement, the predictions underestimate the tunneling effect, which may be attributed to the modification of void ratio to maintain the 'normally-consolidated' state in the calculations. A high value of initial void ratio is kept as constant for the loose sand, while the stress condition increases and the overconsolidation ratio decreases with depth. As required in the critical state model (overconsolidation ratio is not less than 1.0), the initial void ratio for deeper soil is modified based on the normal compression curve, leading to a denser state of sand.

516 The pile capacity degradation curves are provided in Fig. 14g-i, and the critical values of tunnel volume loss using the three criteria presented earlier are indicated (also given in Table 2). These results indicate the empirical pile capacity criterion (i.e. $R_{Q}^{f}=0.85$ ) gives the best fit to the experimental results for the loose sand tests, however this is not an ideal outcome since $R_{Q}^{f}$ does not take into account the initial pile safety factor, which clearly has an effect.

For loose sands, the predictions of initial soil states and model parameters become difficult, and the actual failure mechanisms might be different to those implied within the proposed analytical model, leading to the inconsistency of experimental and analytical results of tunneling induced pile settlement. On the other hand, it is also difficult to prepare a uniform loose sample in centrifuge tests, and disturbance due to model preparation and centrifuge spin-up could lead to some inconsistencies in the experimental data. Despite the differences between experimental and analytical data, the results indicate that the suitability of the analytical method in loose sands requires improvement, either in terms of the applied soil model or the adopted model parameters.

Experimental data of medium dense sand tests with $D_{R} \approx 76 \%$ (i.e. $e_{0} \approx 0.709$ ) were reported by Jacobsz (2002); Table 3 provides details of the centrifuge tests with information on pile depth, horizontal offset, and initial safety factor. The predictions of ground settlement, pile settlement and pile capacity degradation are shown in Fig. 15. The analytical predictions of pile 
settlement are generally satisfactory. The pile capacity criterion (when $R_{Q}^{f}=0.85$ ) is overly conservative in this case, whereas critical values of tunnel volume loss based on pile settlement and safety factor criteria are slightly higher than the experimental values.

\section{Summary}

The experimental and analytical results from all tests (including dense, medium-dense, and loose) where the piles are located relatively close to the tunnel illustrate that the tunneling induced pile settlement is considerably larger than the greenfield ground settlement, indicating that the pile settlement is strongly affected by the degradation of pile capacity.

For the scenario of tunneling under an existing pile, the state of the pile can be assessed in terms of criteria relating to pile capacity, safety factor, and settlement. The suggested cavity expansion-contraction based method has shown its ability to provide a rational approach for obtaining predictions in all these respects. The three criteria can be summarized as follows:

(1) Empirical pile capacity criterion: $R_{Q}^{f} \geq 0.85$;

(2) Safety factor criterion: $R_{Q, S F} \times S F_{0} \geq S F^{f}=1.0$;

(3) Pile settlement criterion: $s^{f} / b_{p} \leq 0.1$ adopted in this study.

The empirical pile capacity criterion is used to determine $V_{l}^{f, R Q}$, which is limited since it does not consider initial pile safety factor, which has an important effect on pile response to tunneling; the method does, however, provide generally conservative predictions. Correspondingly, $V_{l}^{f, S F}$ and $V_{l}^{f, s}$ are obtained according to the safety factor and pile settlement criteria, respectively. The empirical parameters in these criteria could be further refined based on more case study data and sensitivity analyses.

The proposed analytical method outcomes have demonstrated that the pile capacity degradation, mobilized safety factor, and tunneling induced pile settlement can be predicted for the case of 
tunnels excavated beneath single piles with a constant service load. The verification exercise demonstrated that the performance of the analytical predictions was variable; further work is needed to calibrate/modify the approach to achieve better predictions. Nevertheless, the method provides a computationally efficient analytical approach for predicting the critical status and stability of a pile affected by tunneling.

The study presented in this paper is limited to the 2D model presented in Fig. 2, which is assumed to be the critical plane of tunnel-soil-pile interaction. The approach presented here could be extended to consider a three-dimensional analysis to investigate the effects of the excavation face advancement and tunnel lining installation, as well as the coupling effects with pile groups and a superstructure. Moreover, the proposed analytical method requires further verification for undrained analysis of tunneling in clays. It should also be noted that, in the current study, the pile was treated as a rigid body, neglecting compression and deflection during pile installation and tunnel excavation; the proposed analytical method could also be modified to consider these considerations.

The proposed method may be useful within preliminary design stages of new tunnels below existing piles. The suggested analyses can provide guidance to evaluate the influence of the tunnel on pile settlement and performance, help to determine an optimal tunnel plan and profile, or suggest whether additional, more elaborate numerical analyses are warranted. In the risk assessment, the analysis of tunneling induced safety factor for piles can be included within acceptability risk criteria for tunnel construction. Relevant mitigation measures can also be considered to examine the risk reduction effect by using the proposed safety factor criterion.

\section{Conclusions}

Drained solutions of cavity expansion and contraction using the unified clay and sand model, providing stress-strain variation around cavities with large-deformation analyses, were applied in this study to investigate the effect of new tunnel construction in the proximity of an existing pile. Spherical cavity expansion was adopted to estimate the changes of mean stress and specific 
volume in the soil during pile installation. The soil states at the pile tip were then assessed for the prediction of pile end bearing capacity, pile foundation stiffness, and thus the non-linear load-settlement response. A CPT-based design method was used for estimation of pile shaft friction, with consideration of elastic cylindrical cavity expansion caused by shear band dilation around the pile shaft. Tunnel convergence-confinement response was obtained by cylindrical cavity contraction, and the soil deformation was modified based on the singularity and image method for ground loss and ovalization of a shallow tunnel in a semi-infinite medium. The tunnel-soil-pile interaction model was established in a 2D plane by evaluating mean stress and specific volume changes during pile installation and tunnel excavation.

Outcomes from the cavity expansion-contraction based method for tunnel-soil-pile interaction were compared against centrifuge test data using dense, medium dense, and loose sands. Three criteria were proposed for consideration of the pile state: (1) an empirical pile capacity criterion: $R_{Q}^{f} \geq 0.85$; (2) a safety factor criterion: $R_{Q, S F} \times S F_{0} \geq 1.0$; and (3) a pile settlement criterion: $s^{f} / b_{p} \leq 0.1$. These three criteria were used to evaluate critical tunnel volume losses $\left(V_{l}^{f, R Q}, V_{l}^{f, S F}\right.$ and $\left.V_{l}^{f, s}\right)$. The empirical pile capacity criterion does not account for initial pile safety factor, however it is relatively conservative. The safety factor and settlement criteria provide an enhanced evaluation of the pile state, however further work is needed to calibrate/modify the developed methodology to gain more confidence in the outcomes.

\section{Data Availability Statement}

All data, models, and code generated or used during the study appear in the published article.

\section{Acknowledgments}

The authors would like to acknowledge financial supports from the Foundation of Key Laboratory of Transportation Tunnel Engineering (Southwest Jiaotong University), Ministry of 
610 Education (no. TTE2017-04), National Natural Science Foundation of China (no. 51908546),

611 Natural Science Foundation of Jiangsu Province (no. BK20170279), China Postdoctoral

612 Science Foundation (no. 2020T130699), and Jiangsu Planned Projects for Postdoctoral 613 Research Funds (no. 1701196B).

614

615 Supplemental Materials

616 A worked example for TP1-P1 is available online in the ASCE Library (www ascelibrary.org).

617

618 Notation

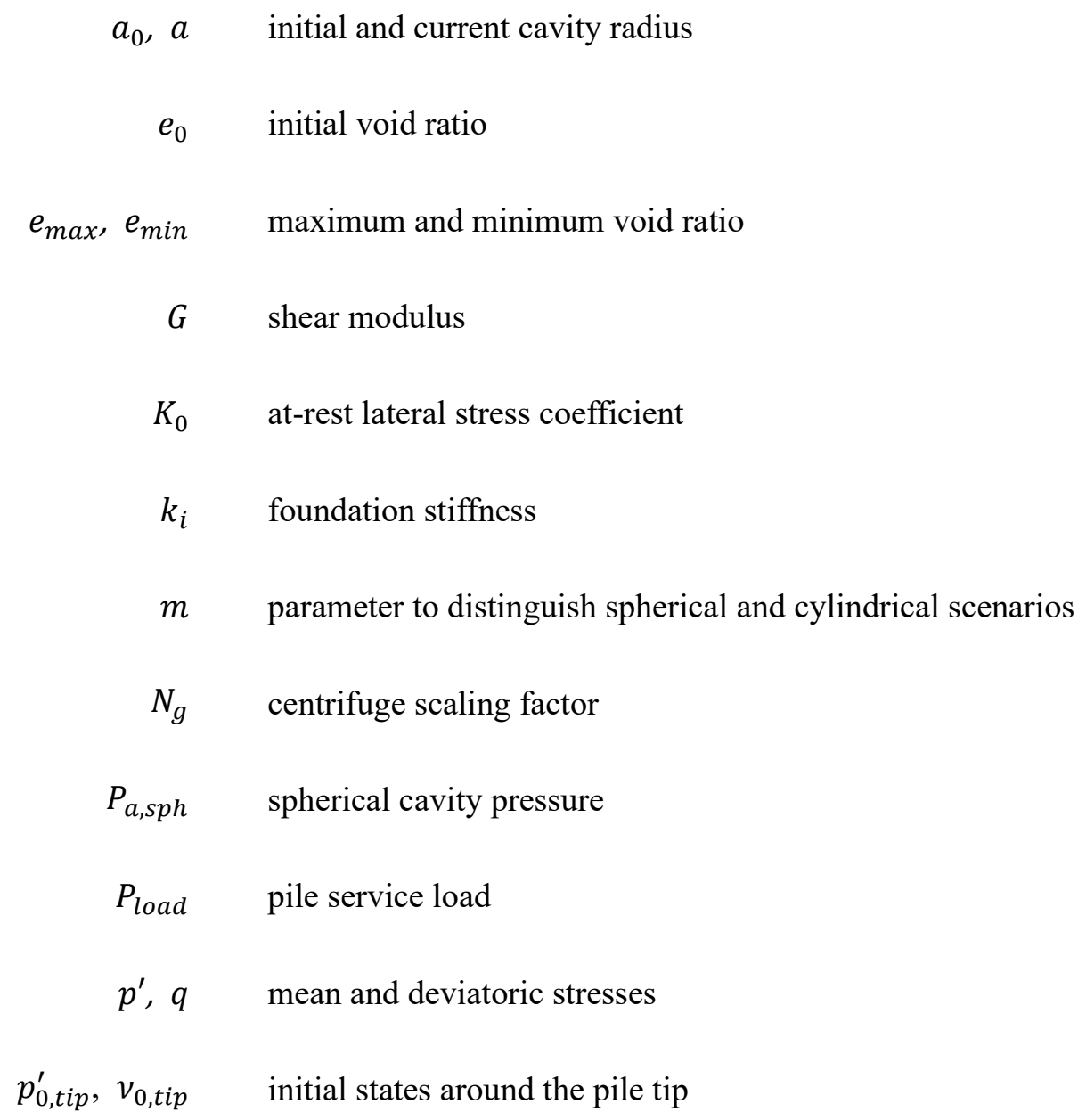


$p_{0, t u n}^{\prime}, v_{0, t u n} \quad$ initial states at depth of tunnel center

$Q \quad$ pile load capacity

$Q_{V l} \quad$ pile load capacity after tunnel volume loss

$q_{c} \quad$ cone tip resistance

$q_{t} \quad$ pile end bearing capacity

$q_{t, V l} \quad$ pile end bearing capacity after tunnel volume loss

$R_{0} \quad$ initial isotropic over-consolidation ratio

$R_{Q} \quad$ reduction factor of pile load capacity

$R_{Q}^{f} \quad$ critical degradation of pile load capacity

$R_{Q, S F} \quad$ critical pile load capacity degradation based on safety factor criterion critical pile load capacity degradation based on pile settlement

$R_{Q, s}$ criterion

$r^{*}, n \quad$ spacing ratio and stress-state coefficient

$r_{p}, b_{p} \quad$ pile radius and pile diameter

$r_{t} \quad$ tunnel radius

$S F_{0} \quad$ initial safety factor

$S F^{f} \quad$ critical safety factor

$S F_{V l} \quad$ safety factor after tunnel volume loss

$s_{\text {pile,Vl } \quad \text { tunneling induced pile settlement }}$

$V_{l, t} \quad$ tunnel volume loss

$V_{l}^{f, e x p} \quad$ critical tunnel volume loss at pile failure based on experimental data 


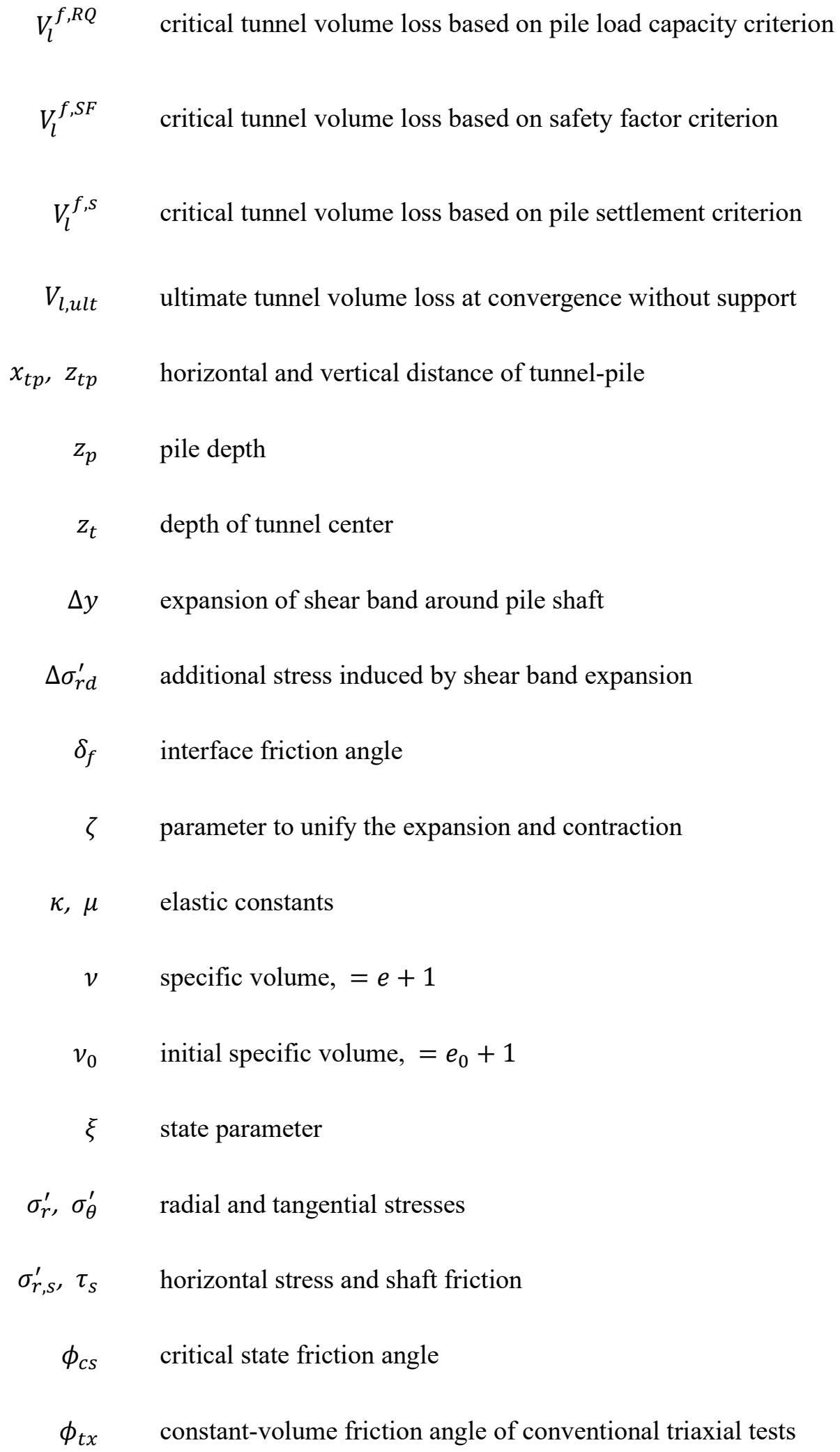


Attewell, P. B., Yeates, J. and Selby, A. R. (1986). Soil movements induced by tunnelling and their effects on pipelines and structures. Blackie and Son Ltd, UK.

Basile, F. (2014). Effects of tunnelling on pile foundations. Soils and Foundations, 54(3), 280295.

Been, K. and Jefferies, M. G. (1985). A state parameter for sands. Geotechnique, 35(2), 99-112.

Burland, J. B., Broms, B. B. and De Mello, V. F. B. (1977). Behaviour of foundations and structures. Proceedings of the 9th International Conference on Soil Mechanics and Foundations Engineering, Tokyo, volume 2, p. 495-546.

Chin, F. K. (1971). Discussion to "Pile tests: Arkansas river project". ASCE Journal of Soil Mechanics and Foundations Division 97(6), 930-932.

Dias, T. G. S. and Bezuijen, A. (2015). Data Analysis of Pile Tunnel Interaction. ASCE Journal of Geotechnical and Geoenvironmental Engineering, 141(12), 04015051.

Dias, T. G. S. and Bezuijen, A. (2018). Pile tunnel interaction: Pile settlement vs Ground settlements. ITA World Tunnel Congress 2018 - The role of underground space in building future sustainable cities, Dubai, United Arab Emirates, May, 2530-2539.

Franza, A. (2016). Tunnelling and its effects on piles and piled structures. $\mathrm{PhD}$ thesis, University of Nottingham.

Franza, A. and Marshall, A. M. (2017). Centrifuge modelling of tunnelling beneath axially loaded displacement and non-displacement piles in sand. Geotechnical Frontiers, T. L. Branson and R. Valentine, eds., Orlando, Florida, ASCE Geotechnical Special Publication $277,576-586$.

Franza, A., Marshall, A. M., Haji, T. K., Abdelatif, A. O., Carbonari, S., \& Morici, M. (2017). A simplified elastic analysis of tunnel-piled structure interaction. Tunneling and Underground Space Technology, 61, 104-121.

Franza, A. and Marshall, A. M. (2018). Centrifuge modelling study of the response of piled 
structures to tunnelling. ASCE Journal of Geotechnical and Geoenvironmental Engineering, 144(2), 04017109.

Franza, A. and Marshall, A. M. (2019). Centrifuge and real-time hybrid testing of tunnelling beneath piles and piled buildings. ASCE Journal of Geotechnical and Geoenvironmental Engineering, 145(3), 04018110.

Franza, A., Marshall, A. M. and Zhou, B. (2019). Greenfield tunnelling in sands: the effects of soil density and relative depth. Geotechnique, 69(4), 297-307.

Gonzalez, C. and Sagaseta, C. (2001). Patterns of soil deformations around tunnels. Application to the extension of Madrid Metro. Computers and Geotechnics, 28, 445-468.

He, C., Jiang, Y. C., Fang, Y., Feng, K. and Wang, J. (2013). Impact of shield tunneling on adjacent pile foundation in sandy cobble strata. Advances in Structural Engineering, 16(8), $1457-1467$.

Hu, N. (2015). On fabric tensor-based constitutive modelling of granular materials: theory and numerical implementation. PhD Thesis, University of Nottingham.

Huang, M., Zhang, C. and Li, Z. (2009). A simplified analysis method for the influence of tunneling on grouped piles. Tunnelling and Underground Space Technology, 24(4), 410422.

Jacobsz, S. W. (2002). The effects of tunnelling on piled foundations. PhD thesis, Cambridge University.

Jacobsz, S. W., Standing, J. R., Mair, R. J., Hagiwara, T. and Sugiyama, T. (2004). Centrifuge modelling of tunnelling near driven piles. Soils and Foundations, 44(1), 49-56.

Jongpradist, P., Kaewsri, T., Sawatparnich, A., Suwansawat, S., Youwai, S., Kongkitkul, W. and Sunitsakul, J. (2013). Development of tunneling influence zones for adjacent pile foundations by numerical analyses. Tunnelling and Underground Space Technology, 34, 96-109. 
Kolymbas, D. (2008). Tunnelling and tunnel mechanics: A rational approach to tunneling, Springer, Berlin.

Ladanyi, B. and Johnston, G. H. (1974). Behaviour of circular footings and plate anchors embedded in permafrost. Canadian Geotechnical Journal, 11, 531-553.

Lehane, B. M., Schneider, J. A. and Xu, X. (2005). The UWA-05 method for prediction of axial capacity of driven piles in sand. International Symposium on Frontiers in Offshore Geotechnics, 683-689.

Loganathan, N. and Poulos, H. G. (1998). Analytical prediction for tunneling-induced ground movements in clays. Journal of Geotechnical and Geoenvironmental Engineering, 124(9), 846-856.

Loganathan, N., Poulos, H. G. and Stewart, D. P. (2000). Centrifuge model testing of tunnelling induced ground and pile deformations. Géotechnique, 50(3), 283-294.

Mair, R. J. (1979). Centrifuge modelling of tunnel construction in soft clay. PhD thesis, Cambridge University.

Mair, R. J. (2008). Tunnelling and geotechnics: New horizons. Geotechnique, 58(9), 695-736.

Marshall, A. M. (2009). Tunnelling in sand and its effect on pipelines and piles. $\mathrm{PhD}$ thesis, Cambridge University.

Marshall, A. M. (2012). Tunnel-pile interaction analysis using cavity expansion methods. ASCE Journal of Geotechnical and Geoenvironmental Engineering, 138(10), 1237-1246.

Marshall, A. M. (2013). Closure to "Tunnel-Pile Interaction Analysis Using Cavity Expansion Methods" by Alec M. Marshall. ASCE Journal of Geotechnical and Geoenvironmental Engineering, 139(11), 2002-2004.

Marshall, A. M. and Haji, T. K. (2015). An analytical study of tunnel-pile interaction. Tunnelling and Underground Space Technology, 45, 43-51.

Marshall, A. M. and Mair, R. J. (2011). Tunneling beneath driven or jacked end-bearing piles 
697

698

699

700

701

702

703

704

705

706

707

708

709

710

711

712

713

714

715

716

717

Marshall, A. M., Farrell, R., Klar, A. and Mair, R. (2012). Tunnels in sands: the effect of size, depth and volume loss on greenfield displacements. Géotechnique, 62(5): 385-399.

Marshall, A. M., Franza, A. and Jacobsz, S. W. (2020). An assessment of the post-tunneling safety factor of piles under drained soil conditions. ASCE Journal of Geotechnical and Geoenvironmental Engineering, 146(9): 04020097. DOI: 10.1061/(ASCE)GT.19435606.0002348 .

Mo, P. Q. and Yu, H. S. (2017a). Undrained cavity expansion analysis in a unified state parameter model for clay and sand. Géotechnique, 67(6), 503-515.

Mo, P. Q. and Yu, H. S. (2017b). Undrained Cavity-Contraction Analysis for Prediction of Soil Behavior around Tunnels. International Journal of Geomechanics, 17(5). DOI: 10.1061/(ASCE)GM.1943-5622.0000816.

Mo, P. Q. and Yu, H. S. (2018). Drained cavity expansion analysis with a unified state parameter model for clay and sand. Canadian Geotechnical Journal, 55, 1029-1040. DOI: 10.1139/cgj-2016-0695.

Mroueh, H. and Shahrour, I. (2002). Three-dimensional finite element analysis of the interaction between tunnelling and pile foundations. Int. J. Numer. Anal. Methods Geomech., 26, 217-230.

Peck, R. B. (1969). Deep excavations and tunnelling in soft ground. 7th International Conference on Soil Mechanics and Foundation Engineering, Mexico, p. 225-290.

Randolph, M. F., Dolwin, J. and Beck, R. (1994). Design of driven piles in sand. Geotechnique, 44(3), 427-448.

Sagaseta, C. (1987). Analysis of undrained soil deformation due to ground loss. Geotechnique, 37(3), 301-320.

Schofield, A. N. and Wroth, C. P. (1968). Critical State Soil Mechanics. McGraw-Hill Book 
Co., London.

722

Selemetas, D. (2005). The response of full-scale piles and piled structures to tunnelling. $\mathrm{PhD}$ thesis, Cambridge University.

Strack, O. E. (2002). Analytic Solutions of Elastic Tunneling Problems. PhD thesis, Delft University of Technology.

Suzuki, Y. and Lehane, B. M. (2015). Analysis of CPT end resistance at variable penetration rates using the spherical cavity expansion method in normally consolidated soils. Computers and Geotechnics, 69, 141-152.

Verruijt, A. and Booker, J. R. (1996). Surface settlement due to deformation of a tunnel in an elastic half plane. Geotechnique, 46(4), 753-756.

Vesic, A. S. (1977). Design of pile foundations. Technical report, Transport Research Board.

White, D. J. and Bolton, M. D. (2004). Displacement and strain paths during plane strain model pile installation in sand. Geotechnique, 54(6), 375-397.

White, D. J. and Bolton, M. D. (2005). Comparing CPT and pile base resistance in sand. Proceedings of the Institution of Civil Engineers-Geotechnical Engineering, 158(1), 3-14.

Williamson, M. G. (2014). Tunnelling effects on bored piles in clay. PhD thesis, Cambridge University.

Williamson, M. G., Mair, R. J., Devriendt, M. D. and Elshafie, M. Z. E. B. (2017). Open-face tunnelling effects on non-displacement piles in clay - part $2:$ tunnelling beneath loaded piles and analytical modelling. Géotechnique, 67(11), 1001-1019.

Yu, H. S. (1998). CASM: A unified state parameter model for clay and sand. International Journal for Numerical and Analytical Methods in Geomechanics, 22(8), 621-653.

Yu, H. S., Zhuang, P. Z. and Mo, P. Q. (2019). A unified critical state model for geomaterials with an application to tunnelling. Journal of Rock Mechanics and Geotechnical Engineering. DOI: 10.1016/j.jrmge.2018.09.004. 
Zhang, R., Zheng, J., Pu, H., and Zhang, L. (2011). Analysis of excavation-induced responses of loaded pile foundations considering unloading effect. Tunnelling and Underground Space Technology, 26(2), 320-335.

Zhang, Z. and Zhang, M. (2013). Mechanical effects of tunneling on adjacent pipelines based on Galerkin solution and layered transfer matrix solution. Soils Found., 53(4), 557-568.

751 Zhang, Z., Huang, M., Xu, C., Jiang, Y. and Wang, W. (2018). Simplified solution for tunnelsoil-pile interaction in Pasternak's foundation model. Tunnelling and Underground Space Technology, 78(8): 146-158.

Zhang, Z., Huang, M., Zhang, C., Jiang, K. and Lu, M. (2019). Time-domain analyses for pile 755 deformation induced by adjacent excavation considering influences of viscoelastic mechanism. Tunnelling and Underground Space Technology, 85(3): 392-405.

Zhang, Z., Huang, M., Zhang C., Jiang, K., Wang, Z. and Xi, X. (2020). Complex variable solution for twin tunneling-induced ground movements considering non-uniform convergence pattern. International Journal of Geomechanics, 20(6). DOI: 10.1061/(ASCE)GM.1943-5622.0001700.

761 Zhou, B. (2014). Tunnelling-induced ground displacements in sand. PhD thesis, University of 762 Nottingham. 


\section{Tables}

Table 1. Properties of dense sand centrifuge tests from Marshall (2009) and predicted critical tunnel volume loss

\begin{tabular}{cccccccc}
\hline Test ID & $z_{p}, \mathrm{~mm}$ & $x_{t p}, \mathrm{~mm}$ & $S F_{0}$ & $V_{l}^{f, \exp , \%}$ & $V_{l}^{f, R Q}, \%$ & $V_{l}^{f, S F}, \%$ & $V_{l}^{f, s}, \%$ \\
\hline TP1-P1 & 96 & 0 & 1.65 & 0.92 & 0.80 & 1.20 & 1.04 \\
TP2-P1 & 92 & 61 & 1.64 & 2.40 & 1.51 & $2.13^{*}$ & $1.96^{*}$ \\
TP1-P2 & 91 & 130 & 1.56 & DNF & DNF & DNF & DNF \\
\hline
\end{tabular}

Note: soil relative density $D_{r}=90 \%$; centrifuge scaling factor $N_{g}=75$; pile diameter $b_{p}=$

$12 \mathrm{~mm}$; tunnel radius $r_{t}=31 \mathrm{~mm}$; tunnel axis depth $z_{t}=182 \mathrm{~mm}$; DNF=Did Not Fail at

$$
V_{l, t}=5 \% ; * \text { for estimated value. }
$$
volume loss

\begin{tabular}{ccccccc}
\hline Test ID & $\begin{array}{c}x_{t p}, \\
\mathrm{~mm}\end{array}$ & $S F_{0}$ & $V_{l}^{f, \exp , \%}$ & $V_{l}^{f, R Q}, \%$ & $V_{l}^{f, S F}, \%$ & $V_{l}^{f, s}, \%$ \\
\hline P1SF1.5 & 0 & 1.5 & 0.25 & & 1.50 & 1.26 \\
P1SF2.5 & & 2.5 & 3.4 & & DNF & 3.53 \\
\hline P2SF1.5 & & 1.5 & 1 & & 3.72 & 3.03 \\
P2SF2.5 & 75 & 2.5 & DNF & & DNF & DNF \\
\hline P3SF1.5 & 150 & 1.5 & DNF & & DNF & DNF \\
P3SF2.5 & & 2.5 & DNF & & DNF & DNF \\
\hline
\end{tabular}

Note: Soil relative density $D_{r}=30 \%$; Centrifuge scaling factor $N_{g}=60$; Pile 
diameter $b_{p}=13 \mathrm{~mm}$, pile depth $z_{p}=150 \mathrm{~mm}$; Tunnel radius $r_{t}=45 \mathrm{~mm}$; Tunnel axis depth $z_{t}=225 \mathrm{~mm}$; DNF=Did Not Fail at $V_{l, t}=5 \%$.

771 Table 3. Properties of medium dense sand tests based on centrifuge data after Jacobsz (2002) and predicted critical tunnel volume loss

\begin{tabular}{|c|c|c|c|c|c|c|c|c|}
\hline Test ID & $\begin{array}{l}z_{p} \\
\mathrm{~mm}\end{array}$ & $x_{t p}, \mathrm{~mm}$ & $S F_{0}$ & $D_{r}$ & $V_{l}^{f, \exp }, \%$ & $V_{l}^{f, R Q}, \%$ & $V_{l}^{f, S F}, \%$ & $V_{l}^{f, s}, \%$ \\
\hline \multirow[t]{2}{*}{ SWJ20 } & 200 & & 2.53 & $79 \%$ & 2.20 & 0.85 & 2.97 & 2.71 \\
\hline & & 0 & & & & & & \\
\hline SWJ21 & 225 & & 1.52 & $79 \%$ & 0.70 & 0.41 & 0.81 & 0.75 \\
\hline \multirow[t]{2}{*}{ SWJ01 } & 252 & & 2.27 & $76 \%$ & 1.65 & 0.79 & 3.05 & 2.86 \\
\hline & & 50 & & & & & & \\
\hline SWJ05 & 202 & & 1.60 & $76 \%$ & 1.50 & 1.20 & 2.36 & 2.15 \\
\hline
\end{tabular}

Note: Soil relative density $D_{r} \approx 76 \%-79 \%$; Centrifuge scaling factor $N_{g}=75$; Pile diameter $b_{p}=12 \mathrm{~mm}$; Tunnel radius $r_{t}=30 \mathrm{~mm}$; Tunnel axis depth $z_{t}=286 \mathrm{~mm}$; DNF $=$ Did Not Fail at $V_{l, t}=5 \%$. 


\section{List of Figure Captions}

776 Fig. 1. Results of cavity expansion and contraction: (a,b) stress paths in $q-p^{\prime}$ space; (c,d) specific

Fig. 2. Geometric model of tunnel-soil-pile interaction problem

Fig. 3. Calculation flow chart of tunnel-soil-pile interaction problem

Fig. 4. Soil states after pile installation: (a) mean stress field; (b) change of $v$

782 volume during expansion and contraction; (e) cavity pressure-expansion curves, (f) cavity pressure-contraction curves

\section{Fig. 5. Cavity contraction-based tunnel pressure against tunnel volume loss}

Fig. 6. Changes of soil states with respect to normalized soil movement according to cavity contraction model

Fig. 7. Soil deformation after tunnel excavation: (a) vertical displacement; (b) normalized displacement

Fig. 8. Soil state after pile installation and tunnel excavation: (a) mean stress field; (b) change of specific volume, $v$

Fig. 9. Pile response to tunnel excavation: (a) degradation of pile load-settlement curves; (b) decrease of pile load capacity components with tunnel volume loss (in model scale)

Fig. 10. Results of tunnel-soil-pile interaction: (a) tunneling induced ground settlement; (b) tunneling induced pile settlement; (c) tunneling induced degradation of pile capacity

Fig. 11. Sensitivity study for critical criteria: (a) variations of $R_{Q}, S F$ and $s / b_{p}$ against tunnel 
795 Fig. 12. Changes of mean stress (a-c) and specific volume (d-f) due to pile installation and tunnel excavation: (a,d) TP1-P1; (b,e) TP2-P1; (c,f) TP1-P2

797

798

799

800

801

802

803

804

805

Fig. 13. Comparison of predictions with dense sand experimental data for $x_{t p}=0 \mathrm{~mm}$ (TP1-P1), $61 \mathrm{~mm}$ (TP2-P1), and $130 \mathrm{~mm}$ (TP1-P2): (a) pile settlement; (b) degradation of pile capacity

Fig. 14. Comparison of predictions with centrifuge data for loose sand tests with $x_{t p}=0 \mathrm{~mm}$, $75 \mathrm{~mm}$ and $150 \mathrm{~mm}$ : (a-c) ground settlement; (d-f) pile settlement; (g-i) degradation of pile capacity

Fig. 15. Comparison of predictions with centrifuge data for medium dense sand tests with $x_{t p}=$ $0 \mathrm{~mm}$ and $50 \mathrm{~mm}$ : (a-b) ground settlement; (c-d) pile settlement; (e-f) degradation of pile capacity 

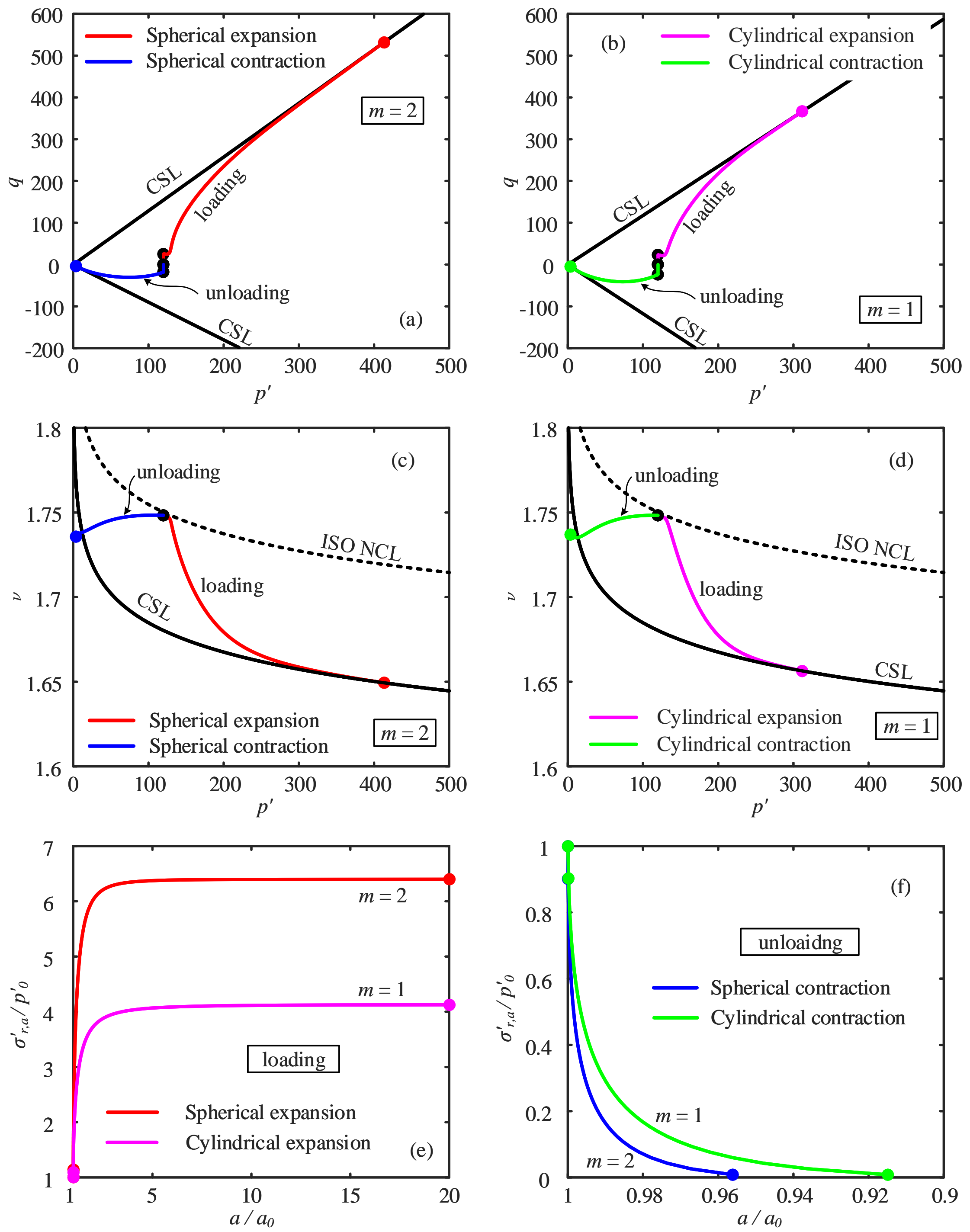
Pile

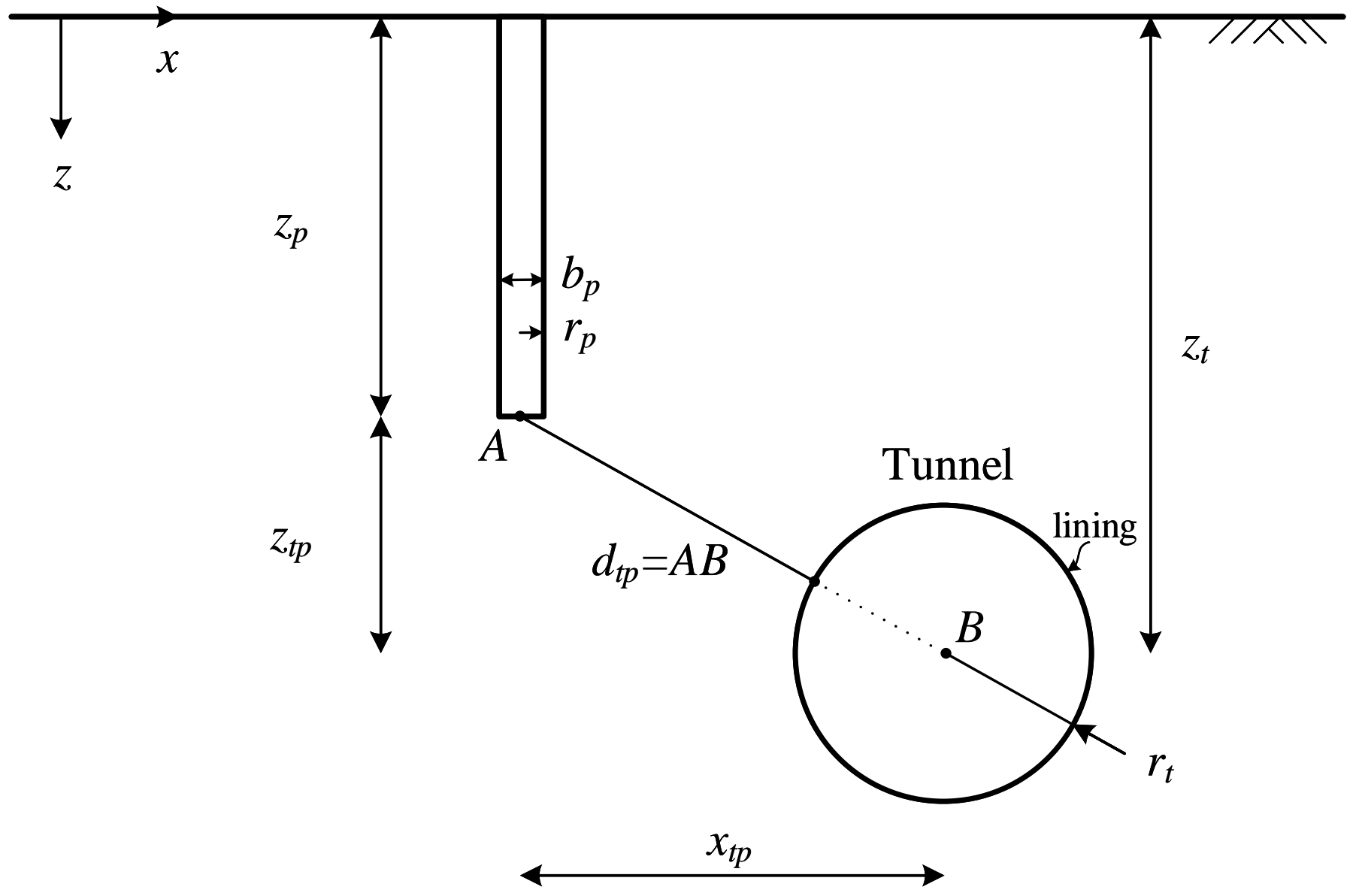




\section{Initial conditions and inputs}

$\downarrow$

1.1 Geometric model

1.2 Soil parameters and initial states

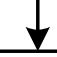

1.3 Initial mean stress and void ratio fields

\section{Pile installation}

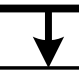

2.1 Tip resistance $q_{c}$ for displacement pile

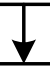

2.2 Shaft friction $\tau_{s}$ for displacement pile

$\downarrow$

2.3 Updated mean stress and void ratio fields

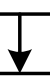

2.4 Pile bearing capacity $\left(Q, Q_{t i p}, Q_{\text {shaft }}\right)$

Zero $p$ / $e$ changes for non-displacement pile

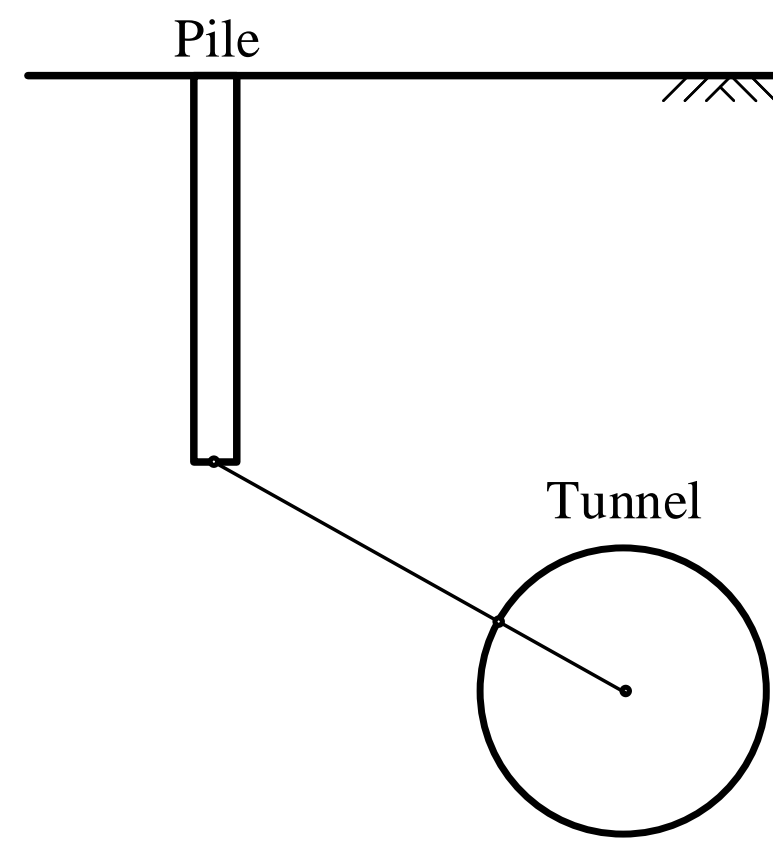

Spherical cavity expansion $a=d_{50} / 2 \rightarrow r_{p}$

CPT-based pile design method UW A-05

Eq. 6

Eq. 8

Spherical cavity expansion $a=r_{p} \rightarrow r_{p} \cdot 110 \%$

\section{Tunnel volume loss}

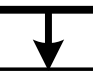

3.1 Tunnel convergence-confinement curve

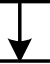

3.2 Updated mean stress and void ratio fields

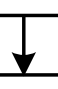

3.3 Reduced pile bearing capacity and $R_{Q}$

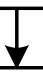

3.4 Tunneling induced settlement
Eq. 10

Eq. 14
Cylindrical cavity contraction

$$
\sigma_{r, a}^{\prime}=p^{\prime}{ }_{0} \rightarrow 1 k P a
$$

Spherical cavity expansion

$$
a=r_{p} \rightarrow r_{p} \cdot 110 \%
$$

Pile load-settlement response hyperbolic asymptote method 
(a) Mean stress field after piling

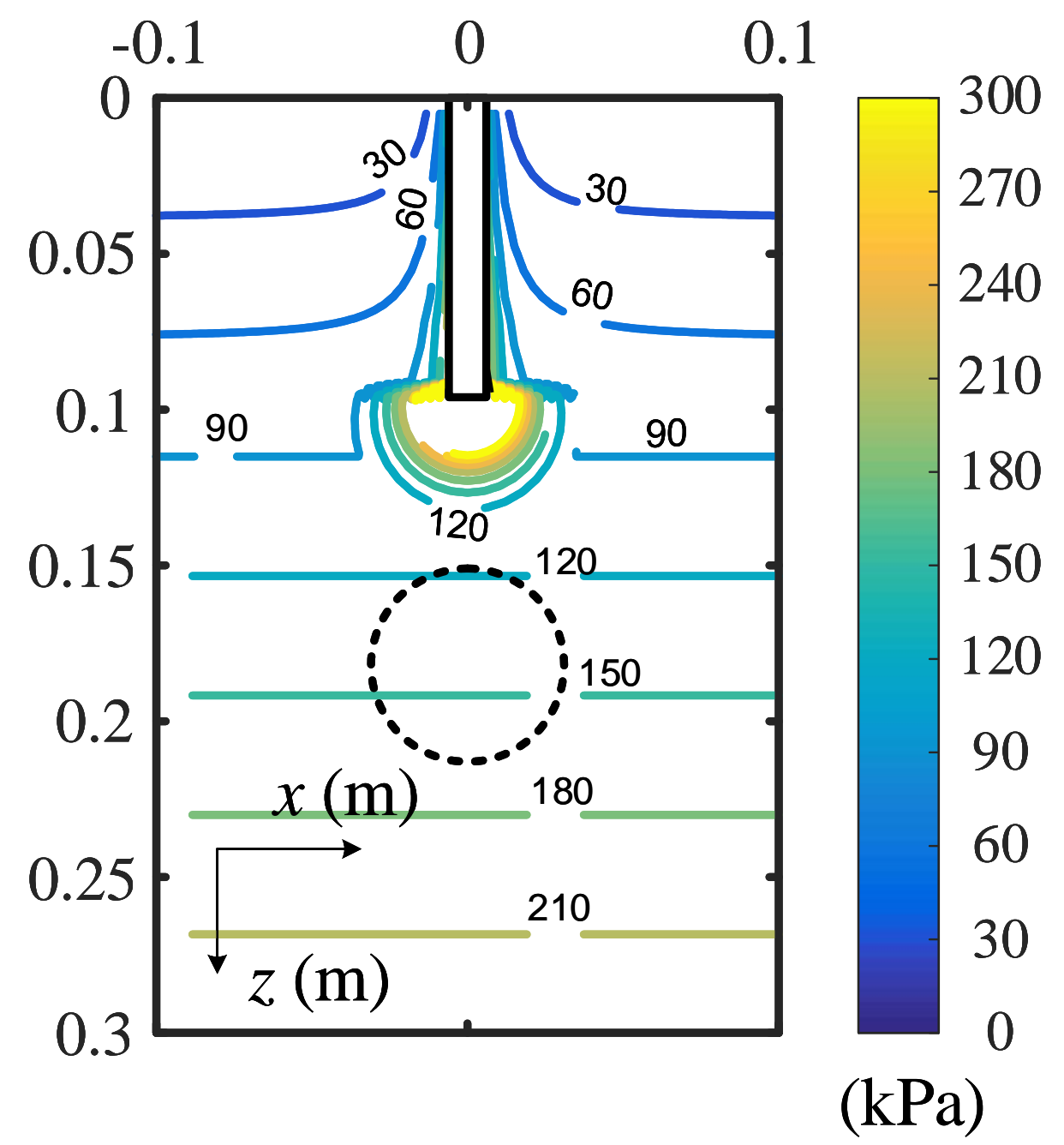

(b) Change of $v$ during piling

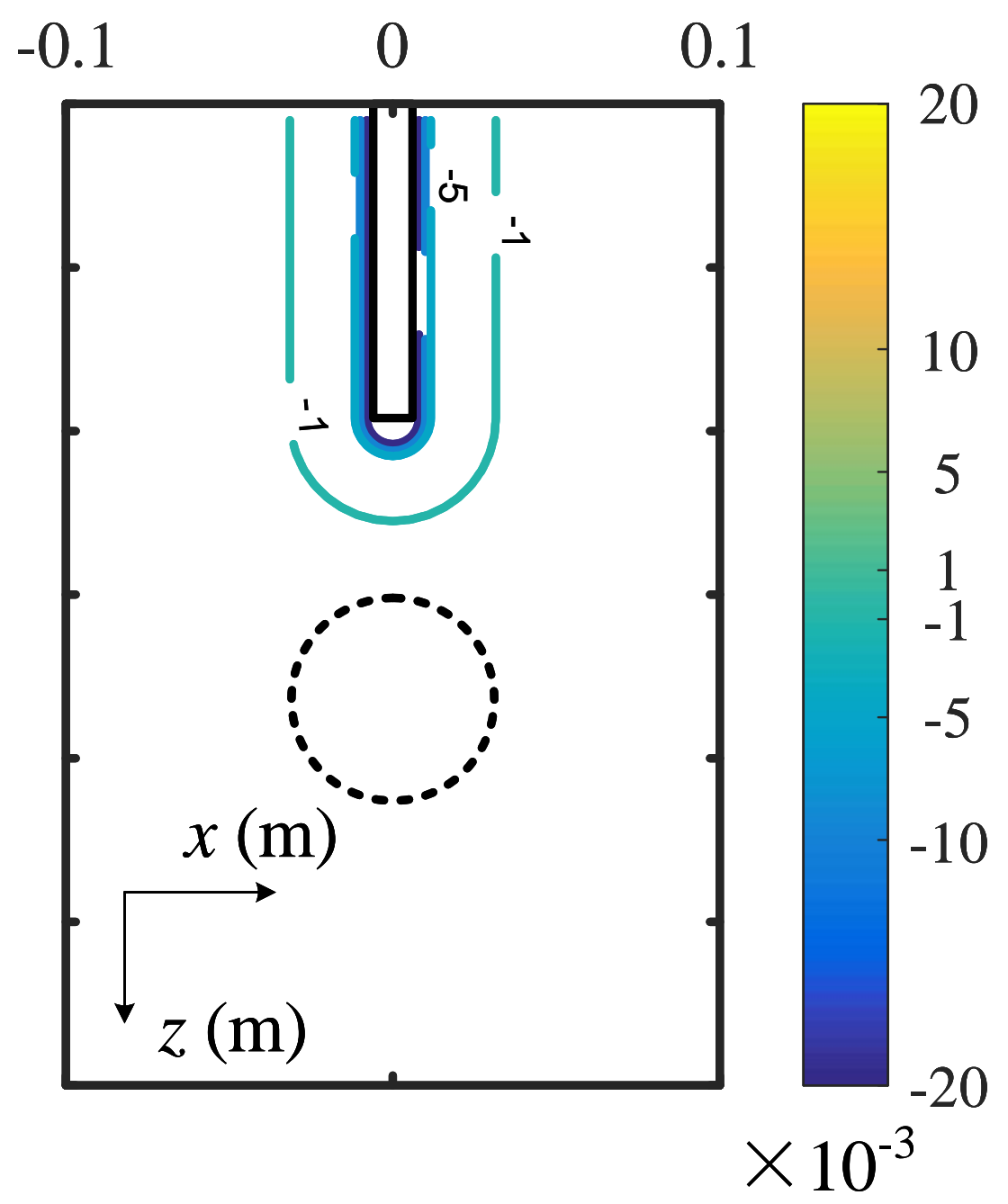




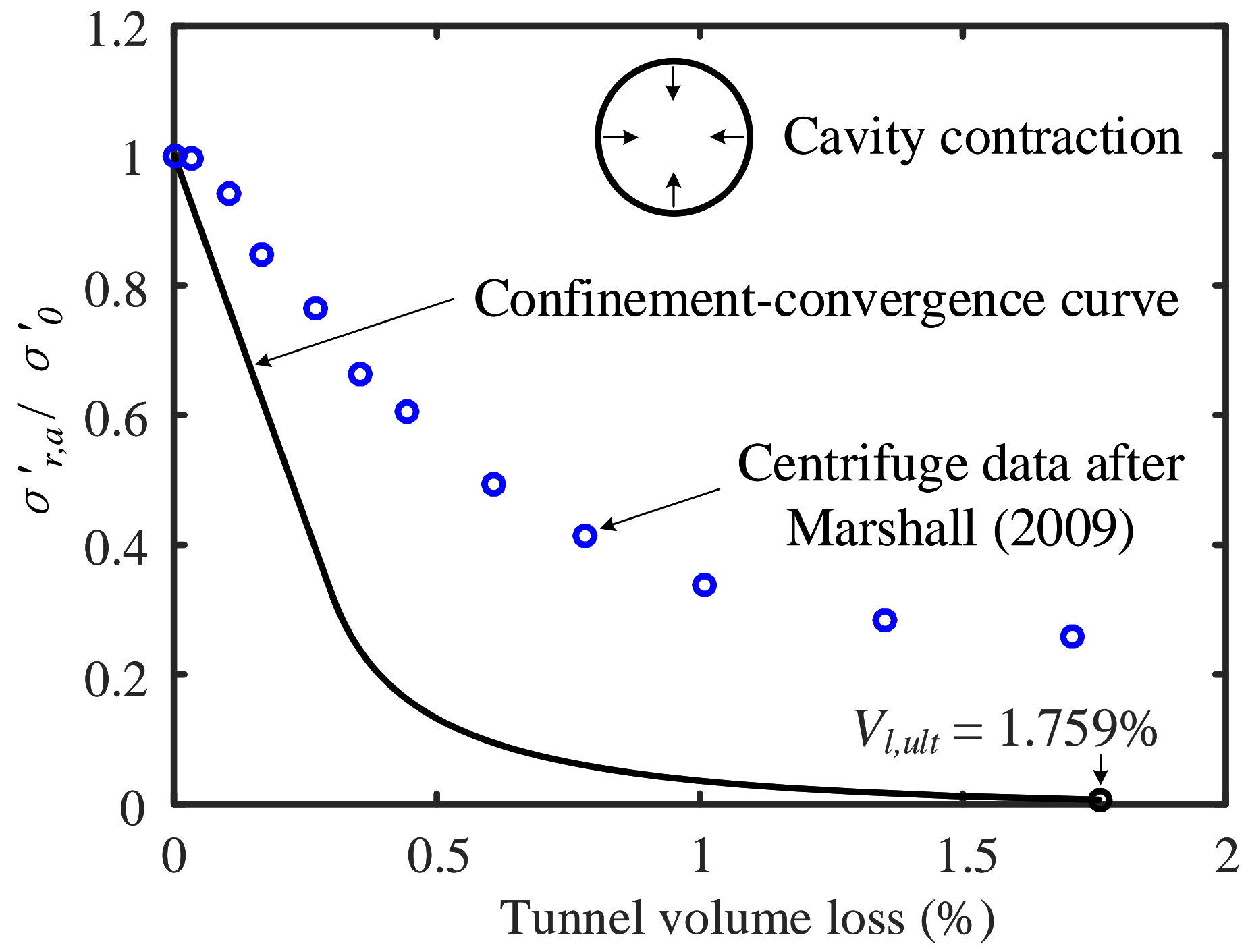



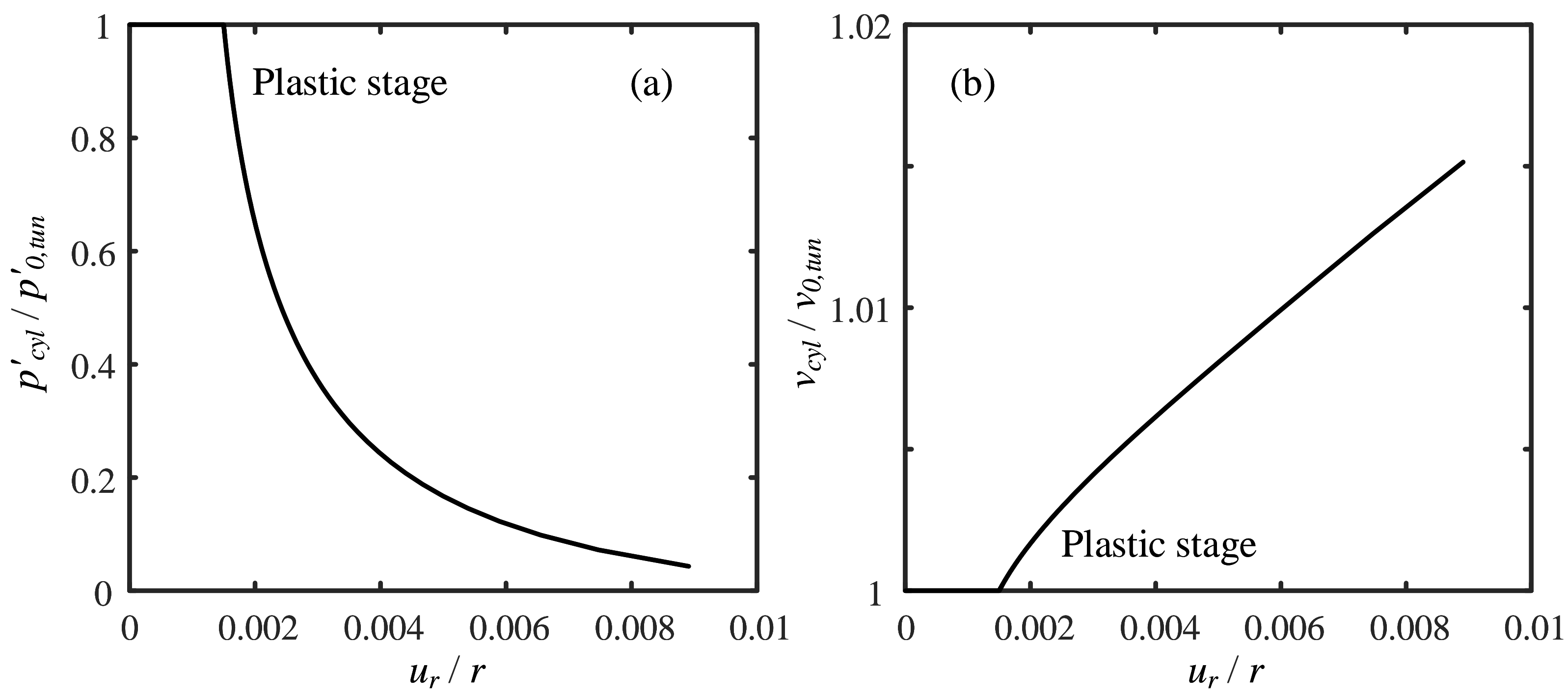
(a) Vertical displacement @ $V_{l, u l t}=1.76 \%$

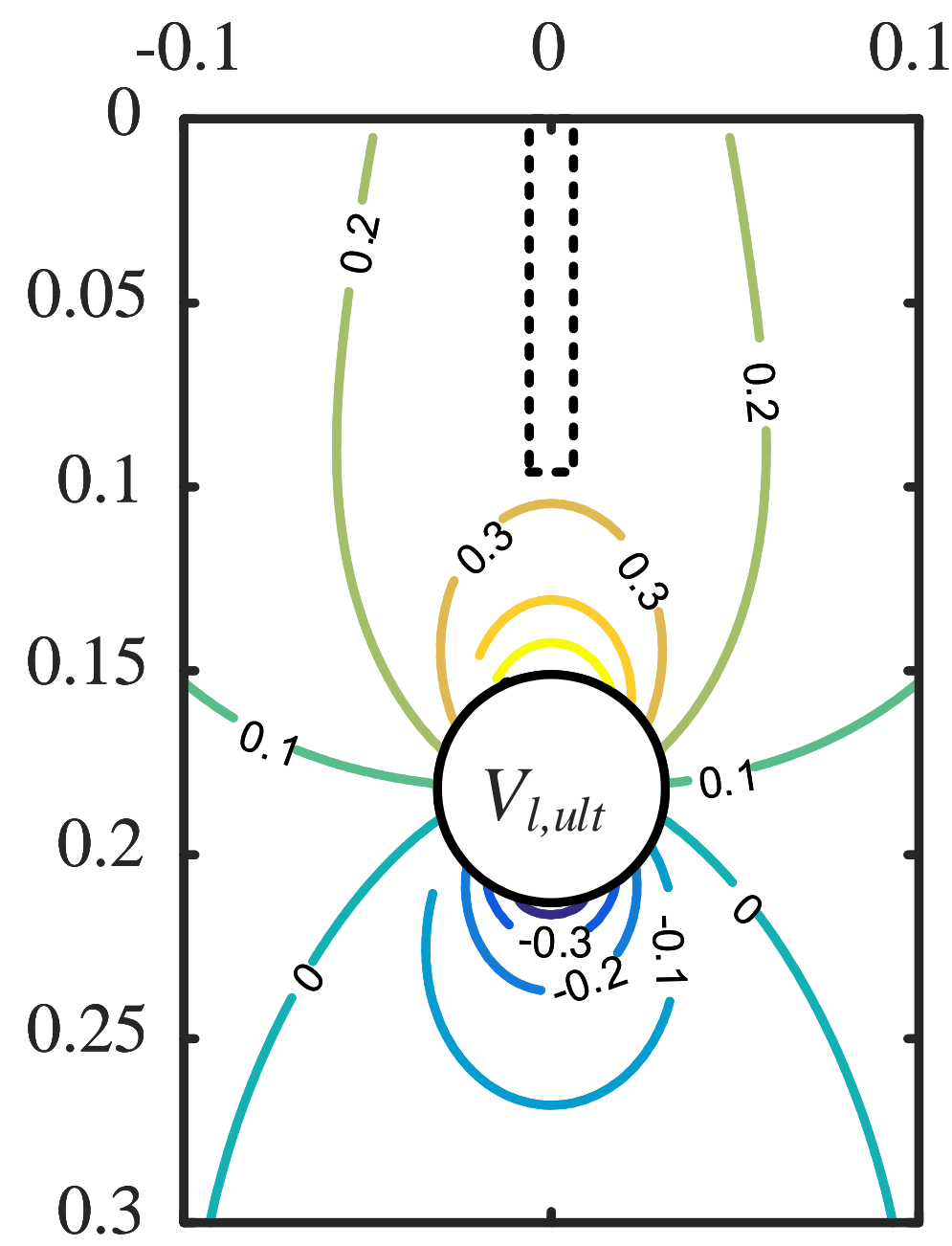

(b) Normalized displacement

@ $V_{l, u l t}=1.76 \%$

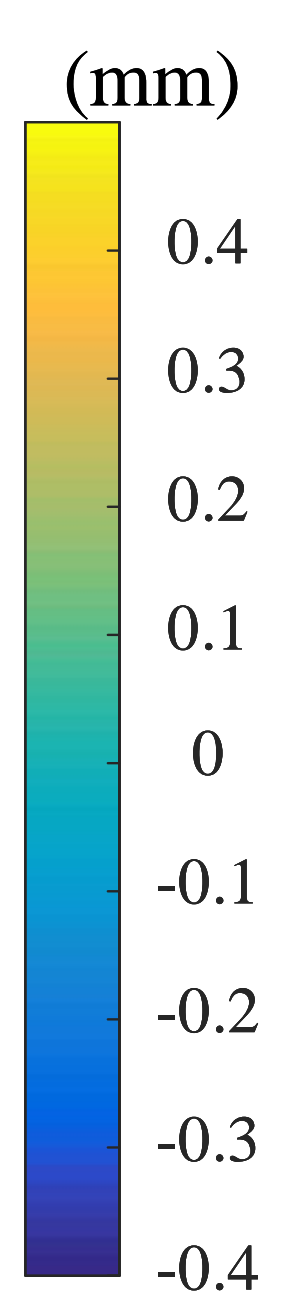

0.018

0.016

0.014

0.012

0.01

0.008

0.006

0.004

0.002 
(a) Mean stress field after piling and tunneling

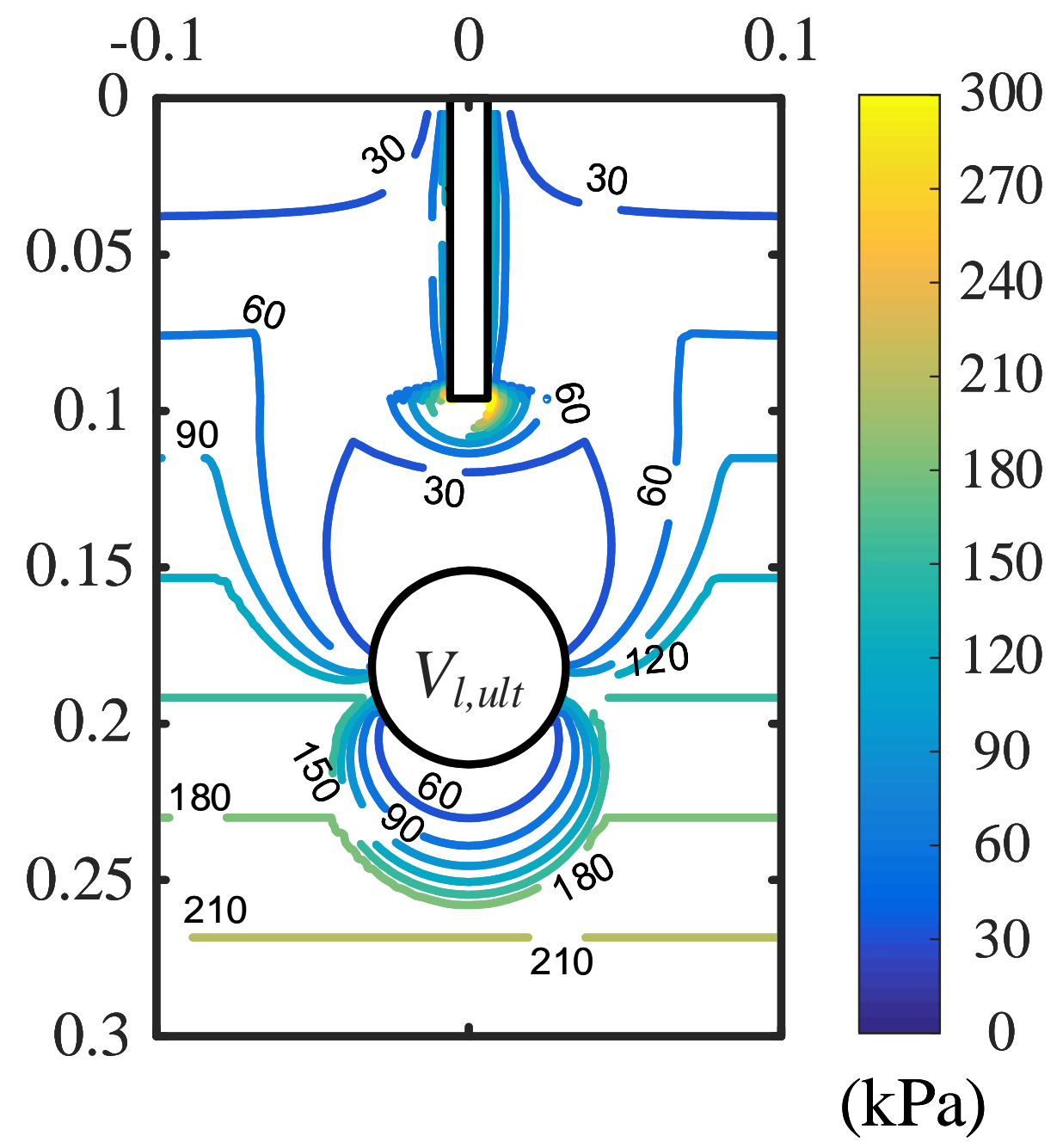

(b) Change of $v$ after piling and tunneling $\begin{array}{lll}-0.1 & 0 & 0.1\end{array}$

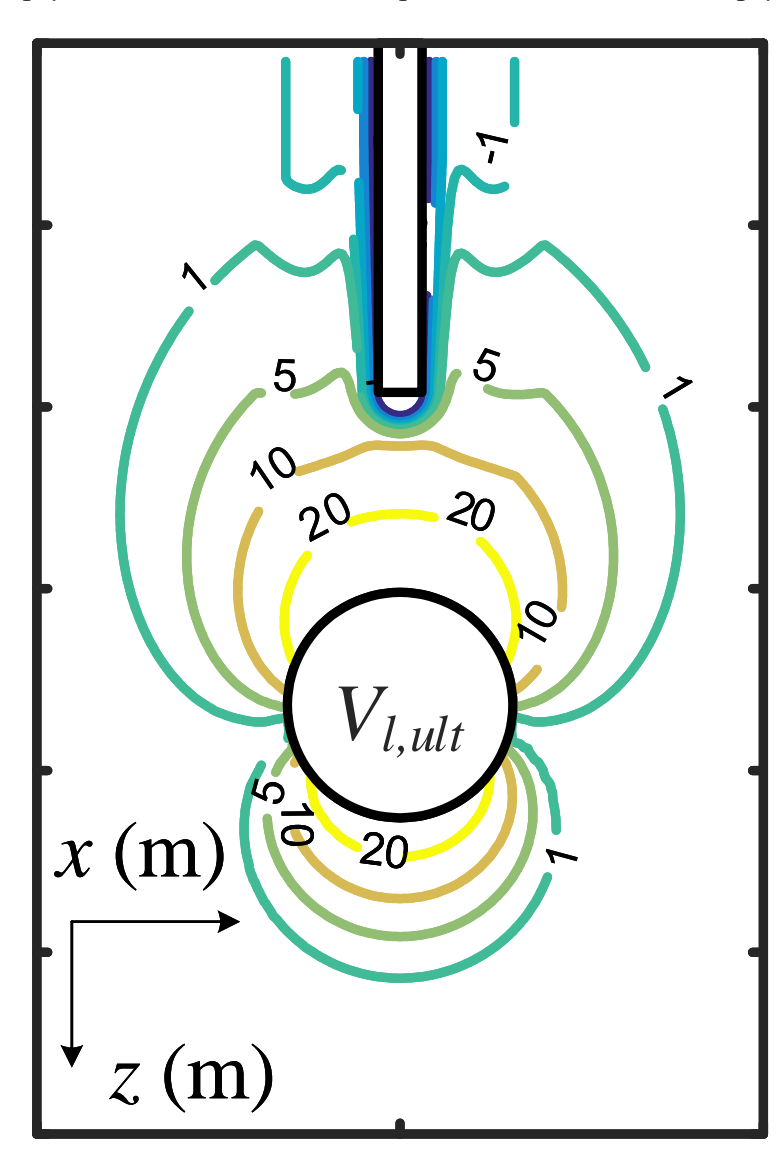



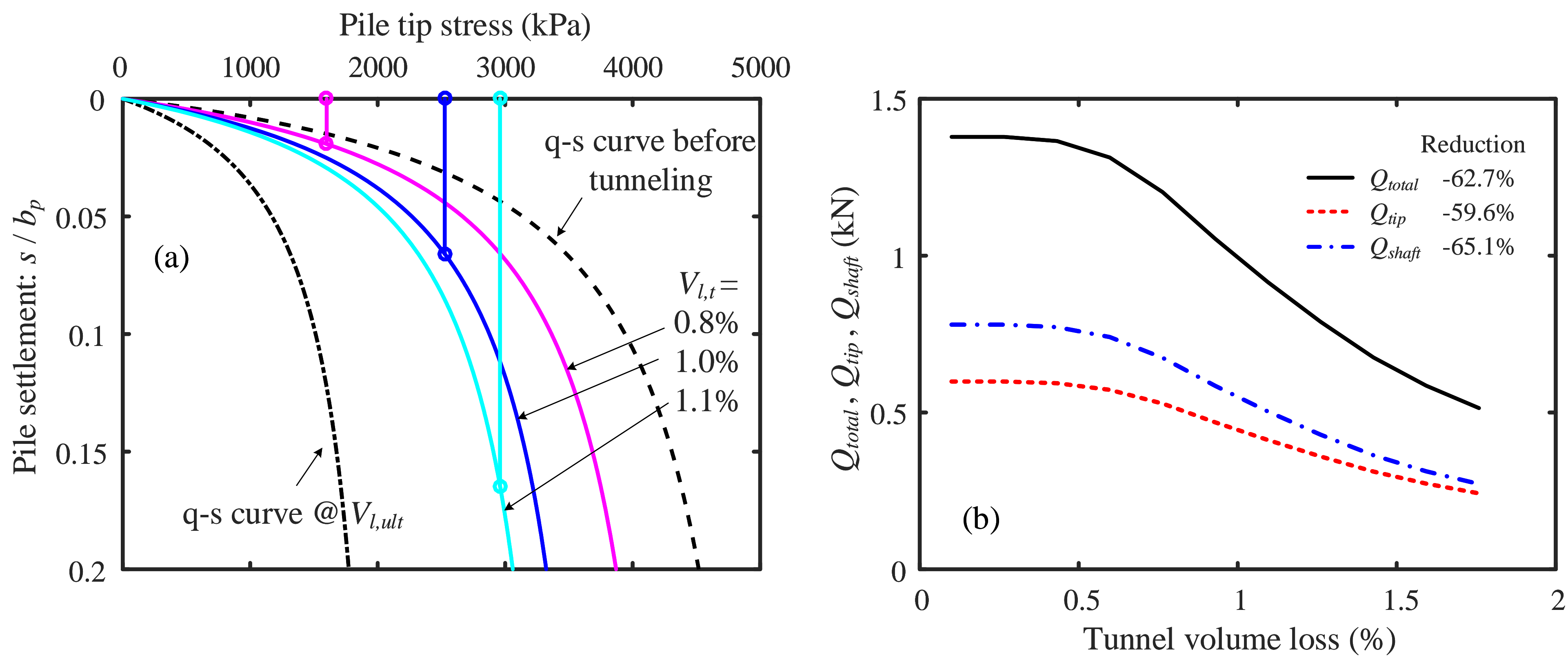

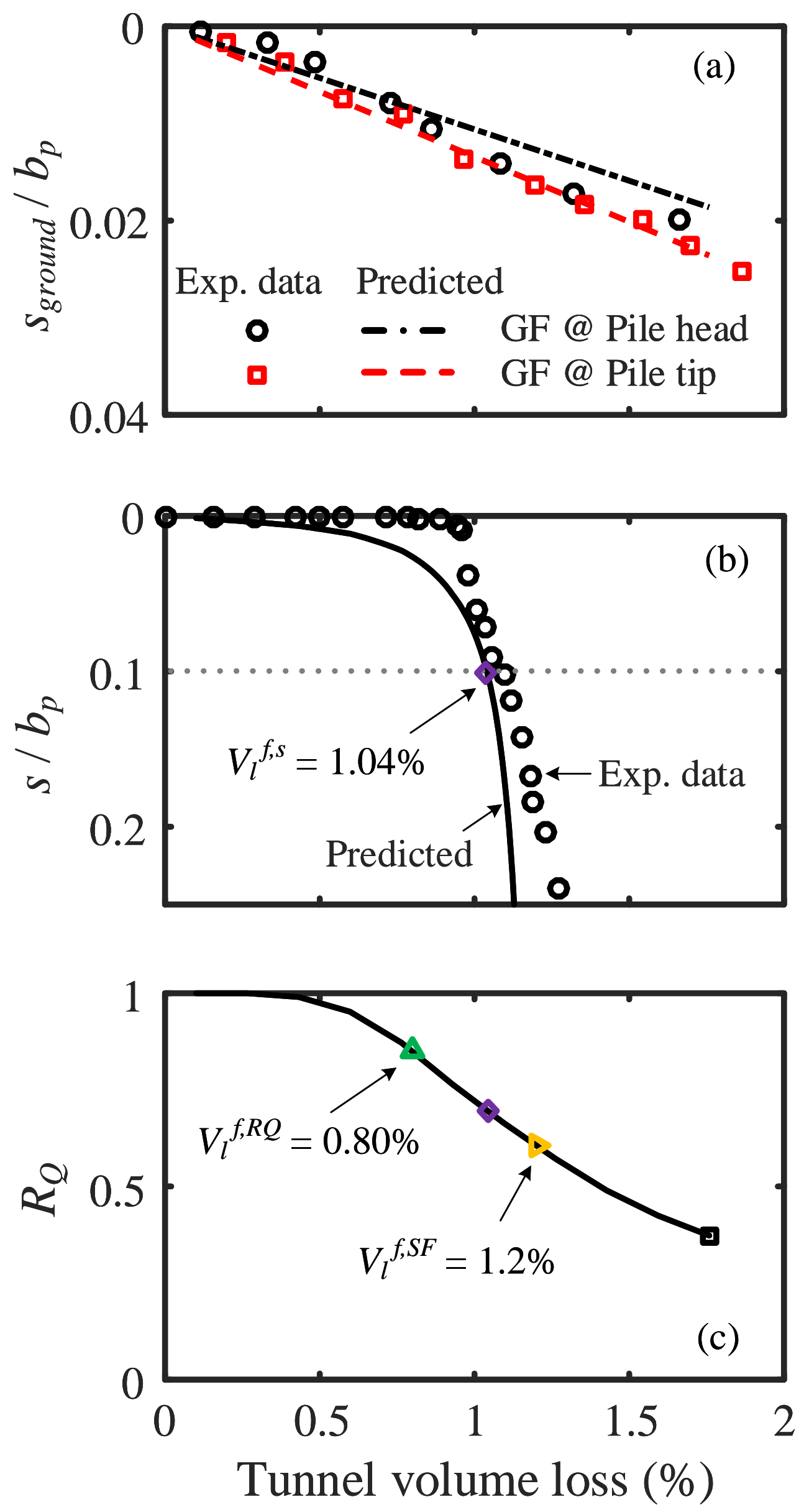


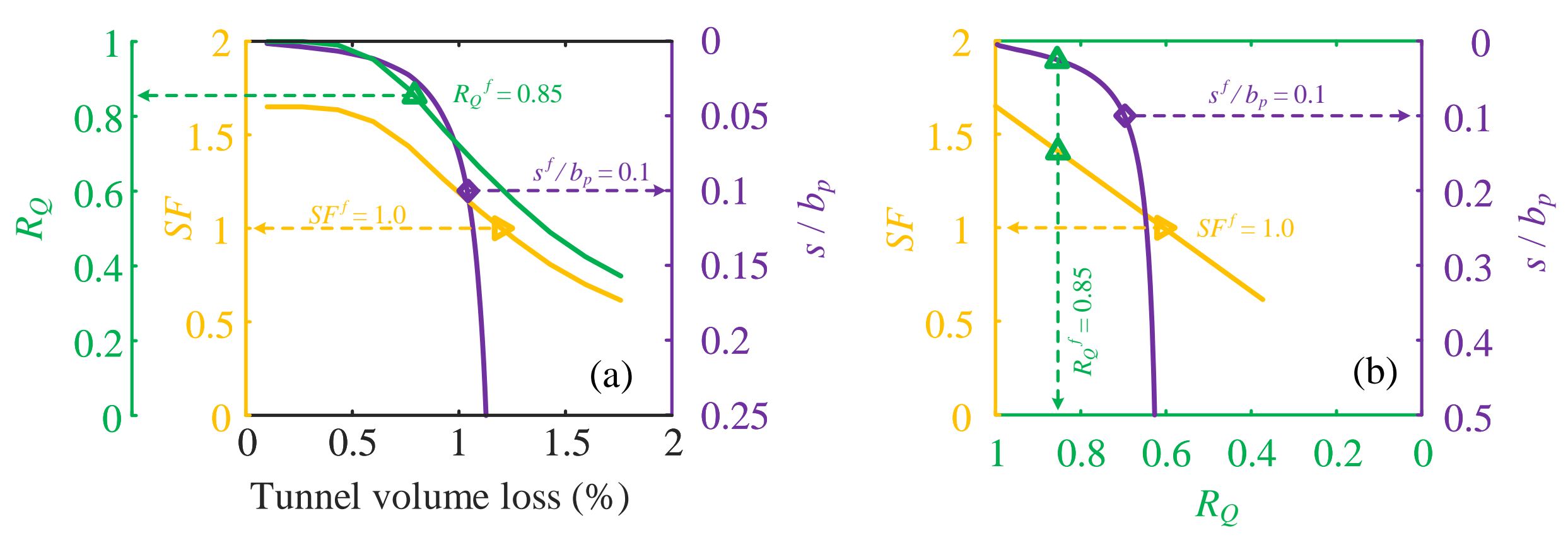




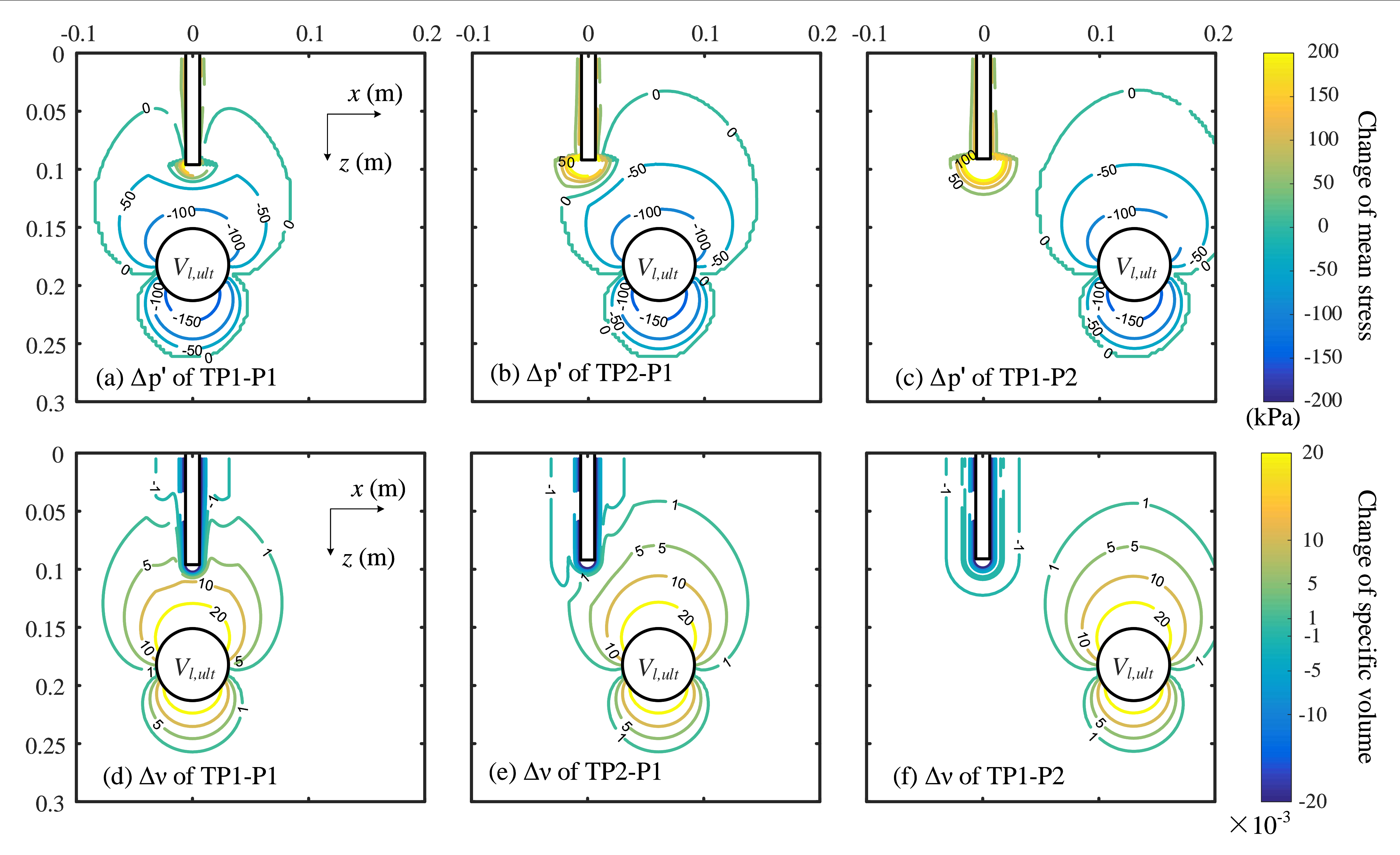



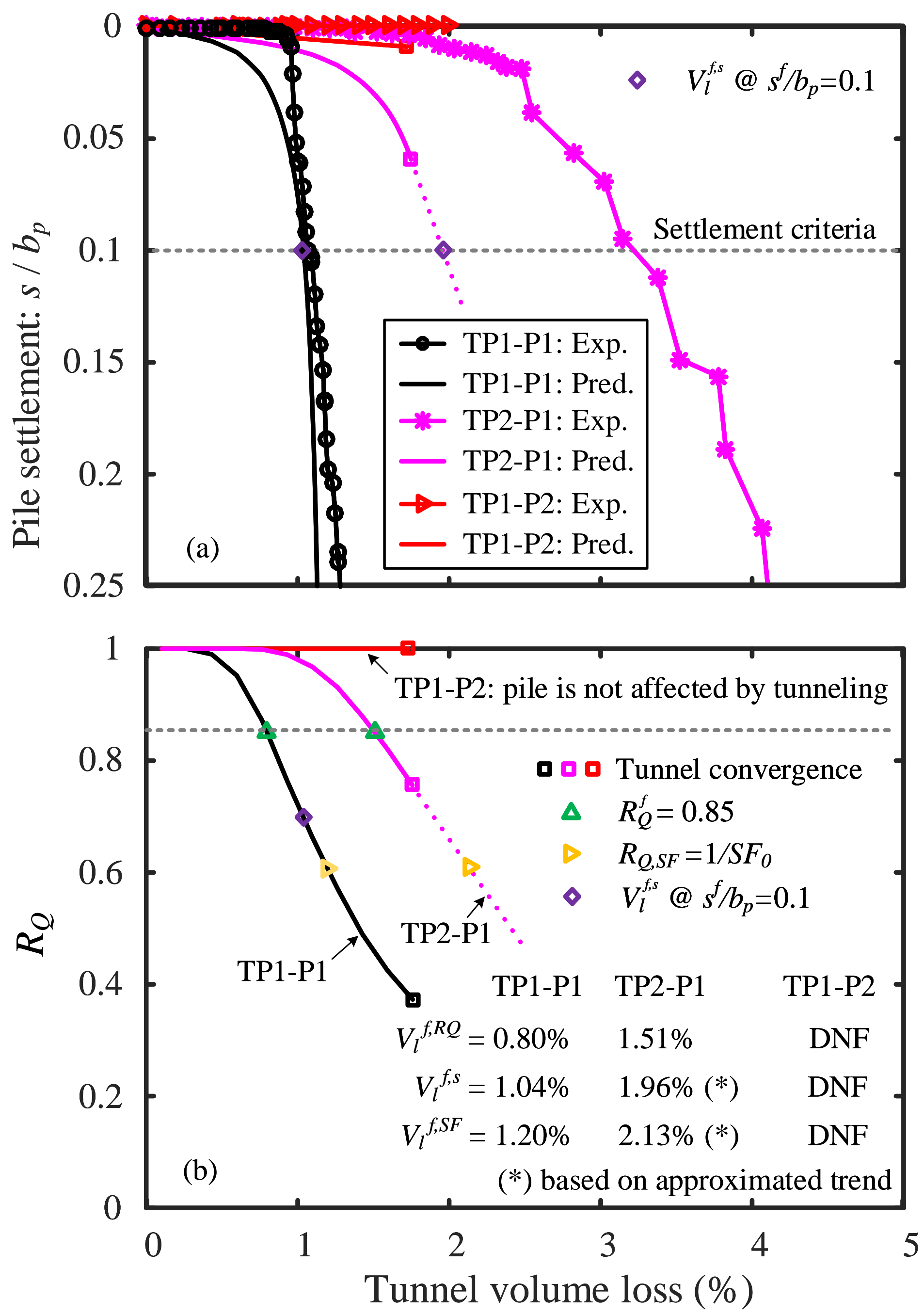


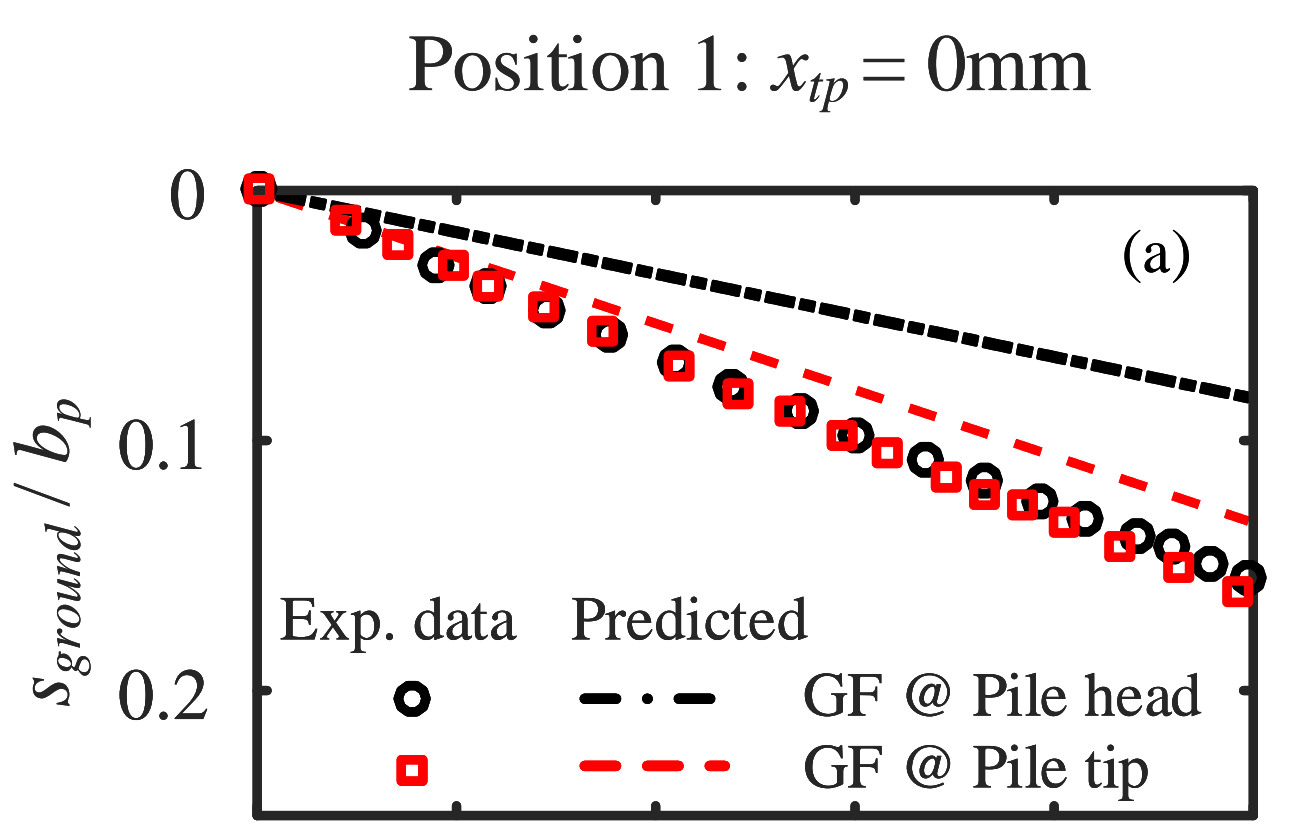

Position 2: $x_{t p}=75 \mathrm{~mm}$
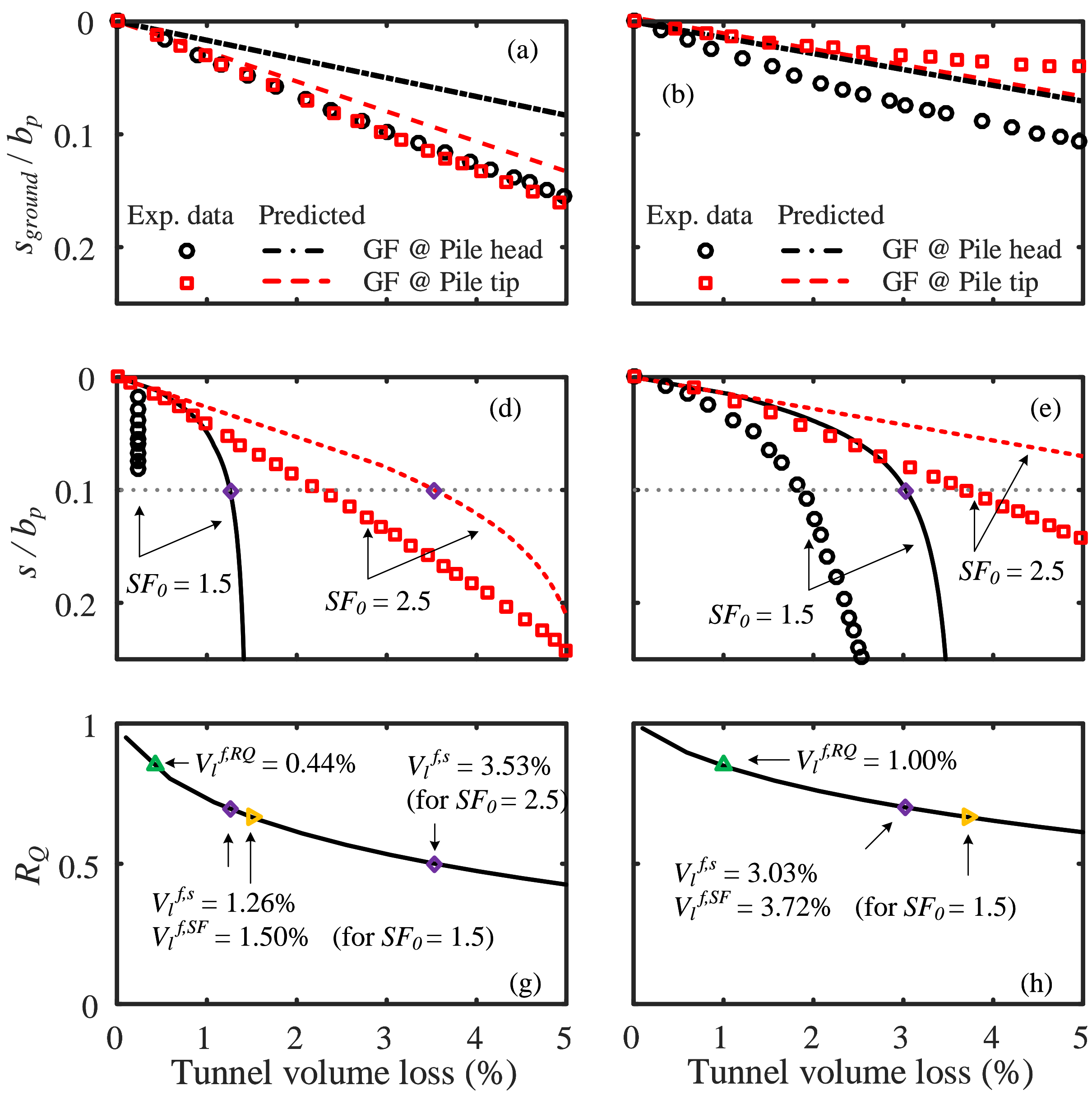
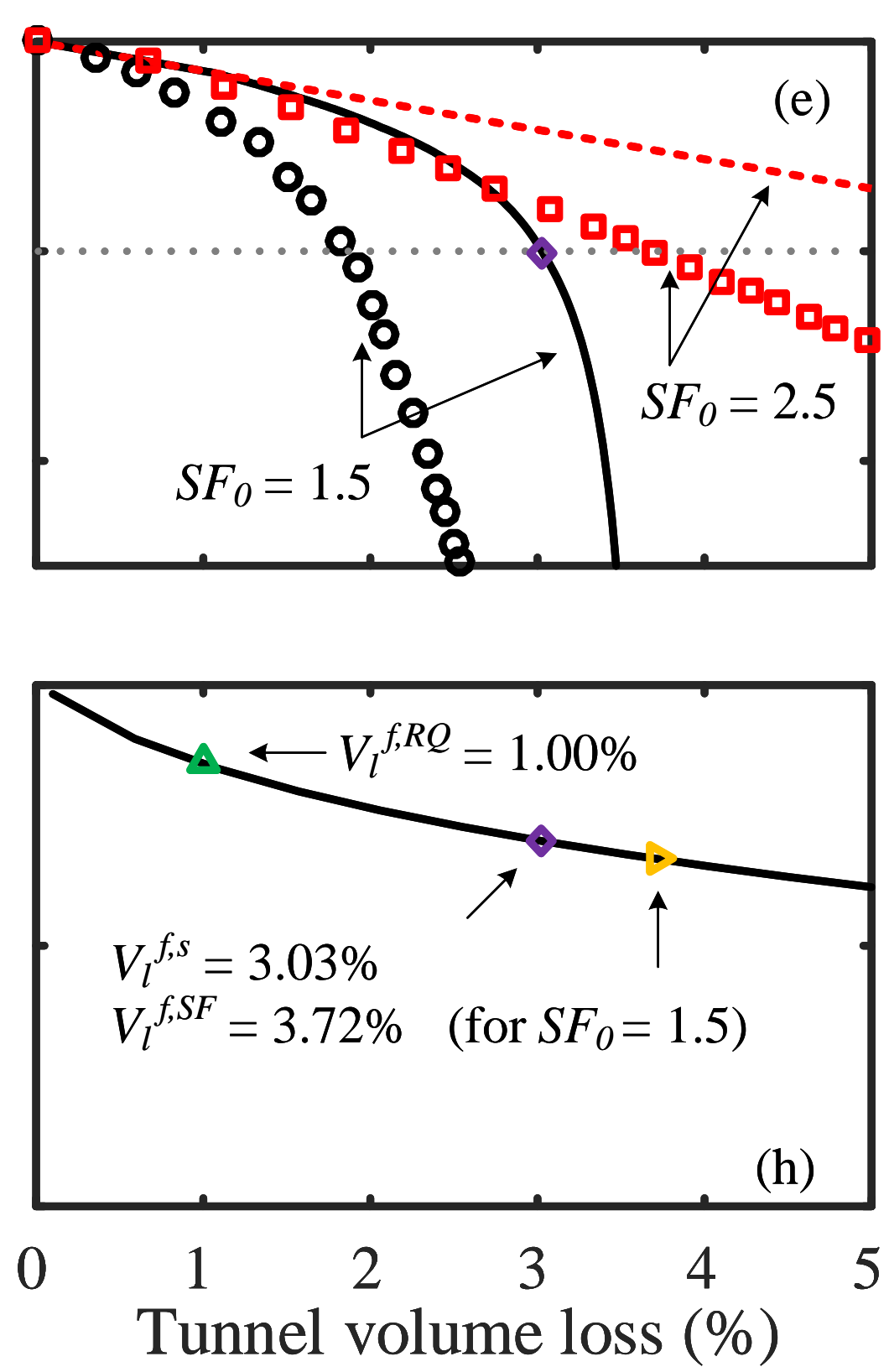

Position 3: $x_{t p}=150 \mathrm{~mm}$
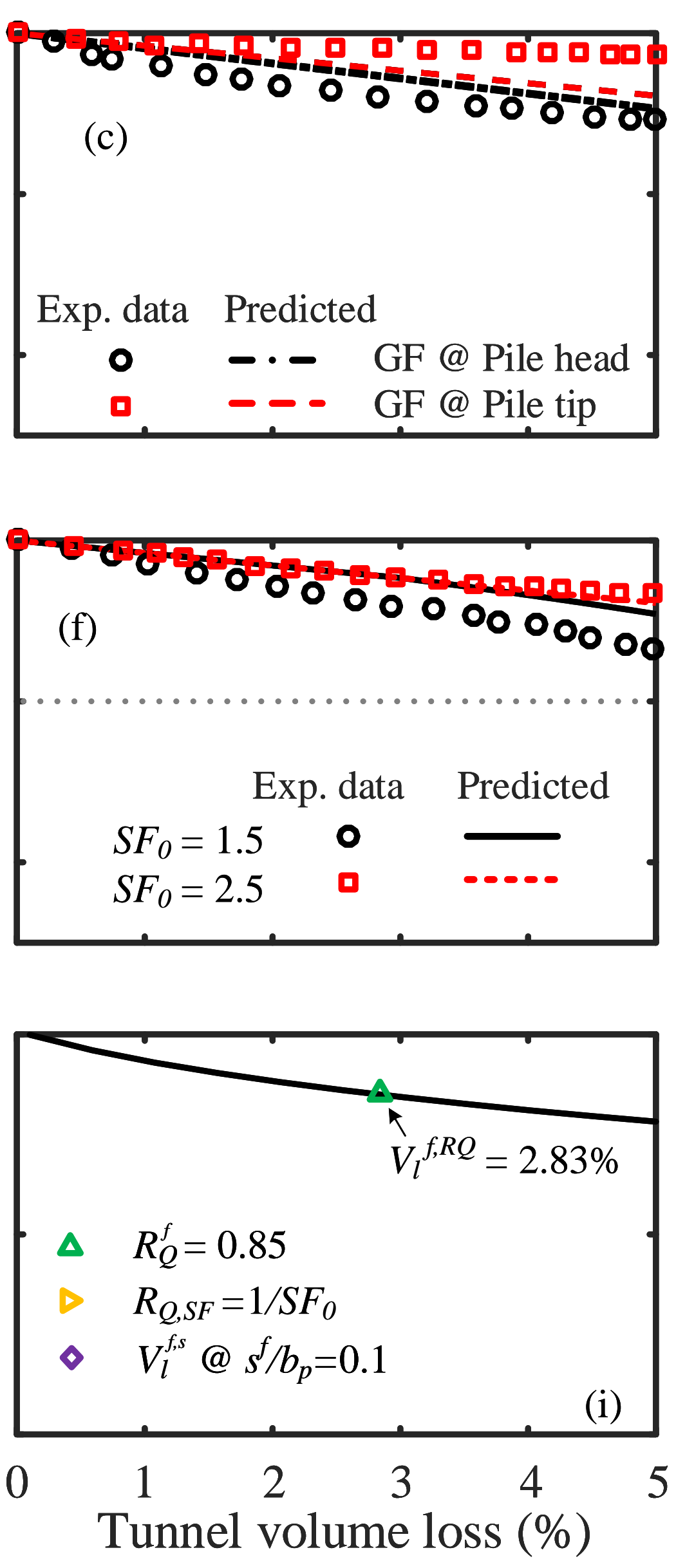


$$
x_{t p}=0 \mathrm{~mm}
$$
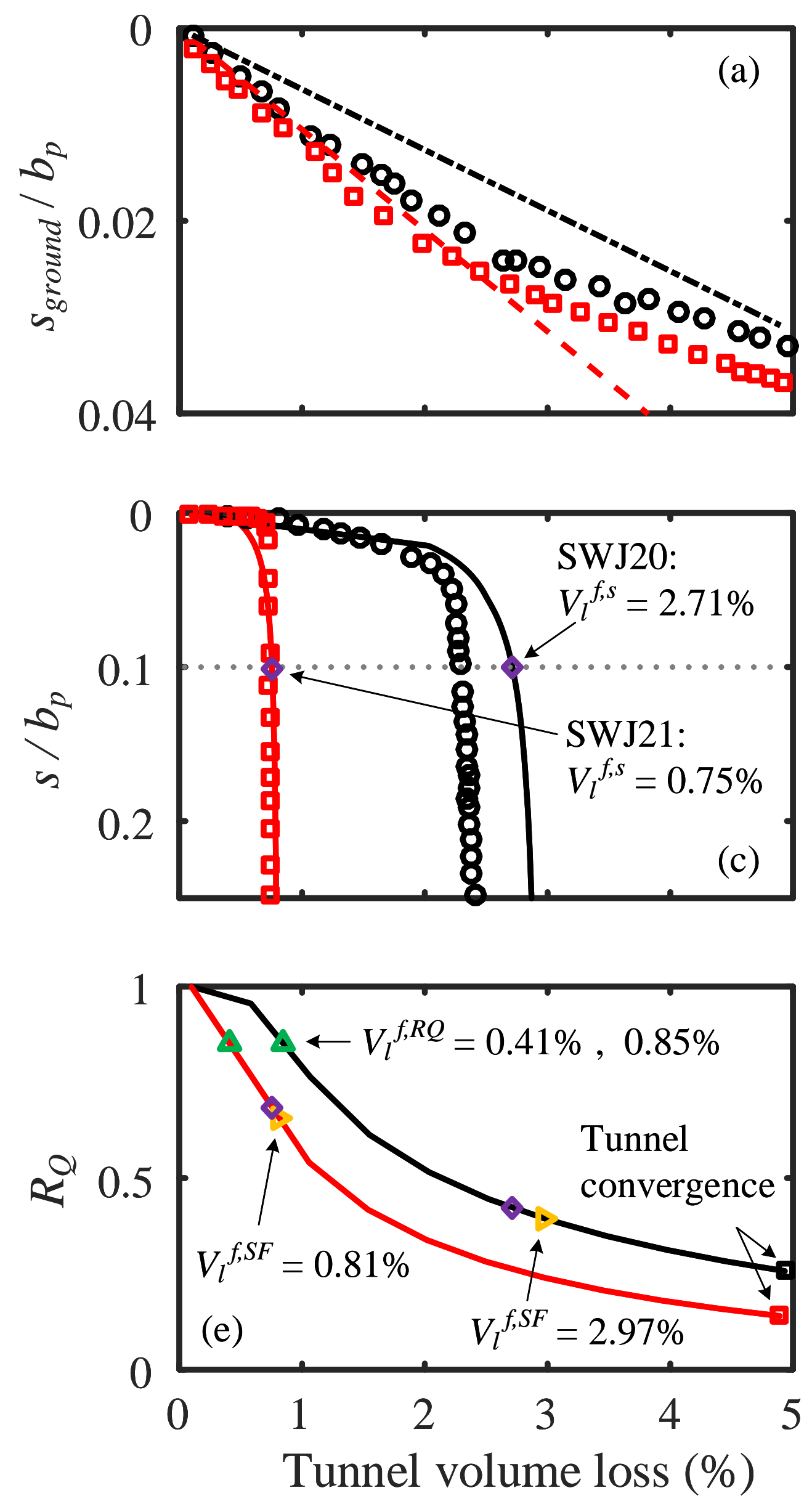

$x_{t p}=50 \mathrm{~mm}$
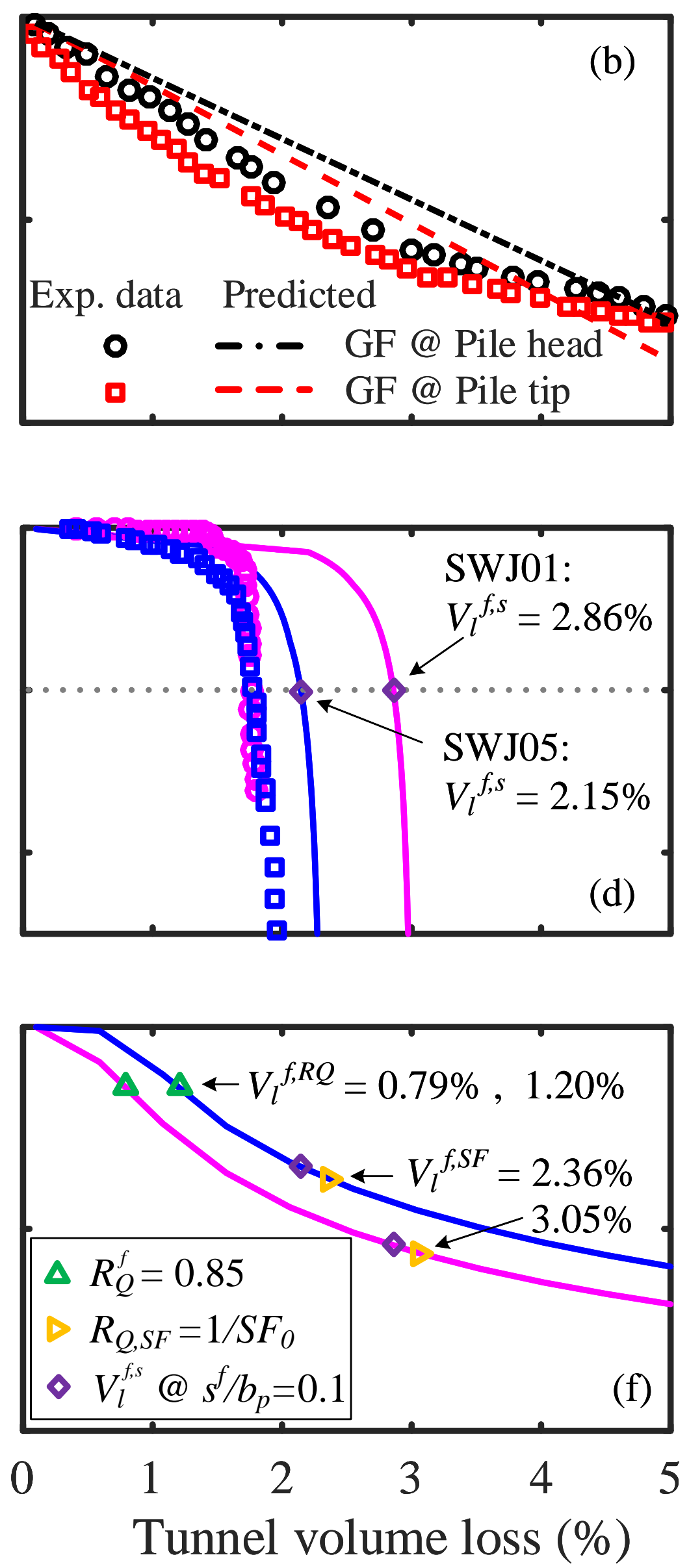


\section{Cavity expansion-contraction based method for tunnel-soil-pile interaction in a} unified clay and sand model: drained analysis

Pin-Qiang Mo, Alec M. Marshall and Yong Fang

\section{Supplementary Materials}

\section{Worked example for TP1-P1}

This supplementary file presents a worked example for TP1-P1, showing the step-by-step calculation for the tunnel-soil-pile interaction problem. The calculation follows the flow chart in Fig. 3 of the main paper.

\section{S1 Initial condition and inputs}

\section{S1.1 Geometric model}

Fig. 2 and Table 1 provide the geometric information of TP1-P1, where the pile is located above the tunnel crown with $x_{t p}=0$. The other geometric parameters include: pile length $z_{p}=$ $96 \mathrm{~mm}$, pile diameter $b_{p}=12 \mathrm{~mm}$; tunnel radius $r_{t}=31 \mathrm{~mm}$, tunnel axis depth $z_{t}=$ $182 \mathrm{~mm}$.

\section{S1.2 Soil parameters and initial states}

Soil parameters in the CASM model are determined in Section 2 for Leighton Buzzard sand, following $\mathrm{Hu}$ (2015): elastic constants $(\kappa=0.005, \mu=0.16)$; critical state constants $(M, \lambda=$ $0.025, \Gamma=1.8)$; CASM constants $\left(r^{*}=33, n=2.0\right) ; \phi_{t x}=32^{\circ}$. The critical stress ratio $M$ is determined by Eq. (1).

The soil initial states include: void ratio and stress condition. (1) Void ratio. For Leighton Buzzard Fraction E sand, the average particle size is $d_{50}=0.122 \mathrm{~mm}, e_{\max }=1.014$ and $e_{\text {min }}=0.613$ (after Franza, 2016). Regarding to the dense sand test of TP1-P1, soil relative 
density $D_{r}=90 \%$, and the initial void ratio is $e_{0}=0.653$. (2) Stress condition. The initial mean stress condition at depth $z$ is $p_{0,0}^{\prime}=\gamma \cdot z \cdot N_{g} \cdot\left(1+2 K_{0}\right) / 3$, where $N_{g}$ is the centrifuge scaling factor with $N_{g}=75$ and $K_{0} \approx 0.5$.

\section{S1.3 Initial mean stress and void ratio fields}

Fig. S1 shows the initial mean stress and void ratio fields.

(a) Initial stress field before piling and tunneling

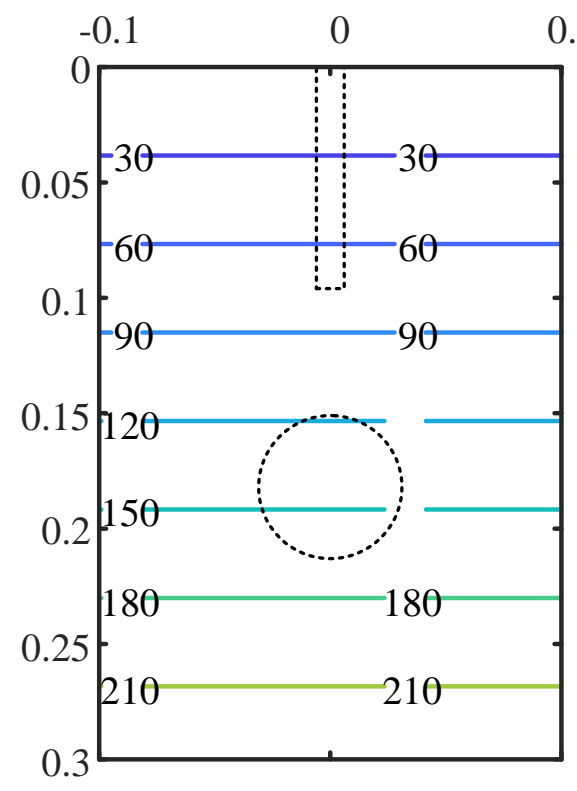

0.1

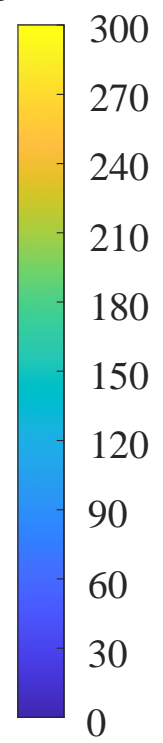

(b) Initial $v$ field before piling $-0.1$

0

0.1

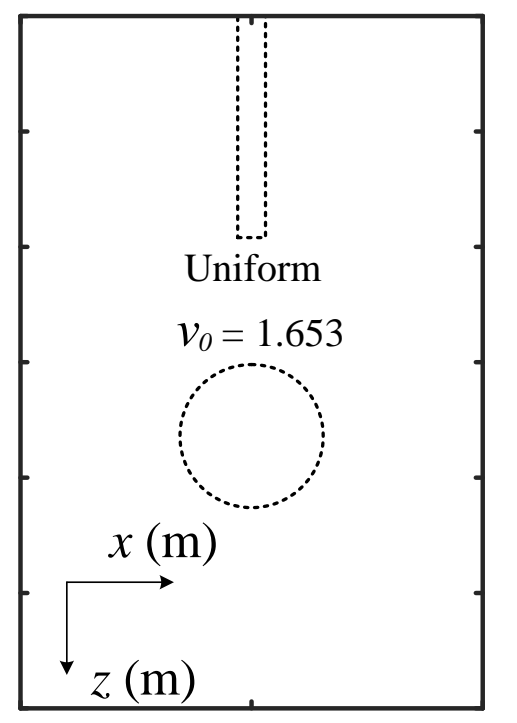

$(\mathrm{kPa})$

Fig. S1 Initial mean stress and specific volume fields before piling

\section{S2 Pile installation}

\section{S2.1 Tip resistance for displacement pile}

When the displacement pile is installed, spherical cavity expansion is used to estimate the penetration resistance and the induced changes around the pile tip with its initial stress condition $p_{0, t i p}^{\prime}=75.1 \mathrm{kPa}$. To represent the pile installation, the spherical cavity with its initial radius $a_{0}=d_{50} / 2$ is expanded to the size of the pile radius $a=r_{p}=b_{p} / 2=6 \mathrm{~mm}$, and the cavity expansion pressure according to Mo and $\mathrm{Yu}$ (2018) is taken to relate to the pile tip resistance during installation based on Eq. (3). In this case, cavity pressure $P_{a, s p h}=1258.9 \mathrm{kPa}$ and tip resistance $q_{c}=4870.9 \mathrm{kPa}$. 


\section{S2.2 Shaft friction for displacement pile}

The normal stress on the pile shaft $\sigma_{r, s}^{\prime}$ and the pile shaft friction $\tau_{\mathrm{s}}$ are estimated by Eq. (4), according to Lehane et al. (2005). The distributions of normal stress and shaft friction along the pile shaft are shown in Fig. S2.

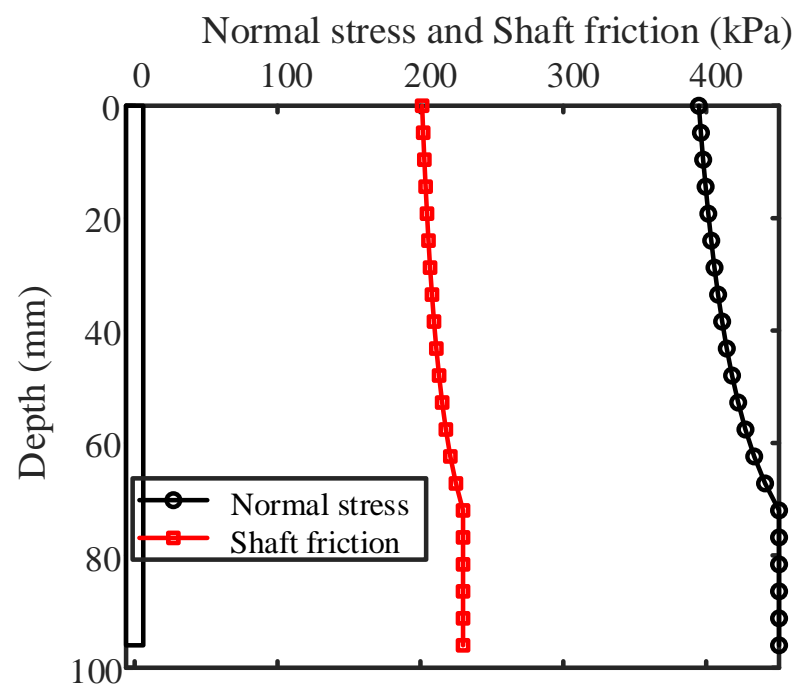

Fig. S2 Distributions of normal stress and shaft friction along the pile shaft

\section{S2.3 Updated mean stress and void ratio fields}

This step calculates the changes of mean stress and void ratio after pile installation. The soil below the pile tip is affected by the spherical cavity expansion, and the soil above the pile tip is estimated using elastic cylindrical cavity expansion.

For soil above the pile tip, the stress distribution at a given depth is obtained from the pattern of elastic cylindrical cavity expansion (i.e. $\left.\sigma_{r}^{\prime}=p_{0}^{\prime}+\left(\sigma_{r, s}^{\prime}-p_{0}^{\prime}\right) \cdot\left(r_{p} / r\right)^{2}\right)$, for estimating the changes of mean stress around the pile shaft, while the void ratio is not changed around the pile shaft. For soil below the pile tip, spherical cavity expansion from Mo and Yu (2018) provides the changes of $p^{\prime}$ and $v$ within the plastic region, which are concentrically distributed. After modification by Eq. (6), the changes $\Delta p^{\prime}$ and $\Delta v$ show reasonable results in Fig. S3(a-b). After pile installation, the updated mean stress and specific volume fields can then be obtained, as shown in Fig. S3(c-d). Note that Fig. S3(b-c) are shown in Fig. 4 of the paper. 
(a) Changes of stress field after piling

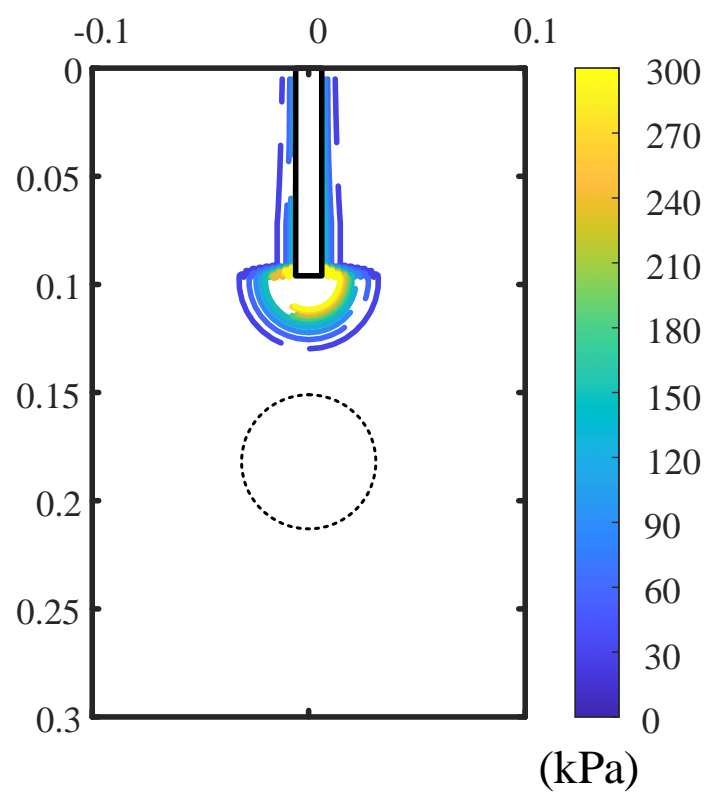

(c) Stress field after piling
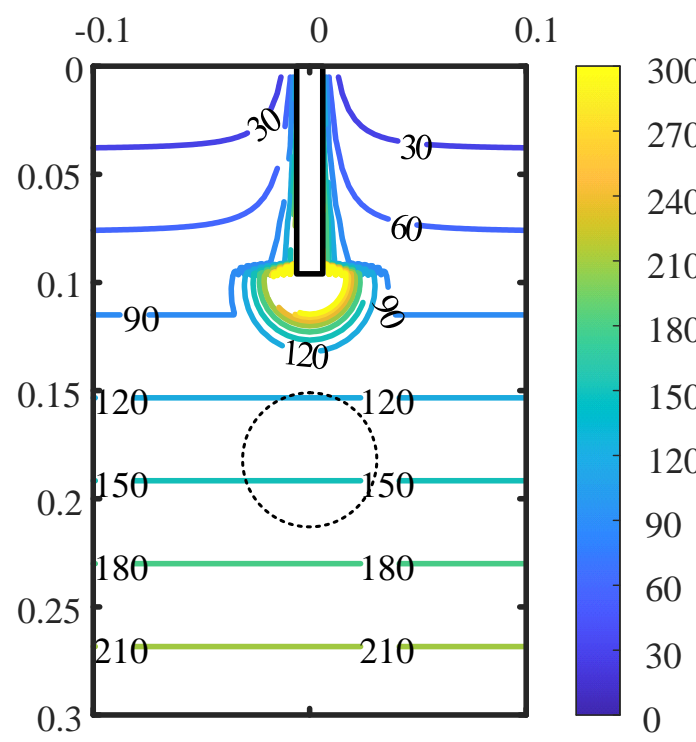

$(\mathrm{kPa})$

\section{S2.4 Pile bearing capacity}

(b) Changes of specific volume field after piling

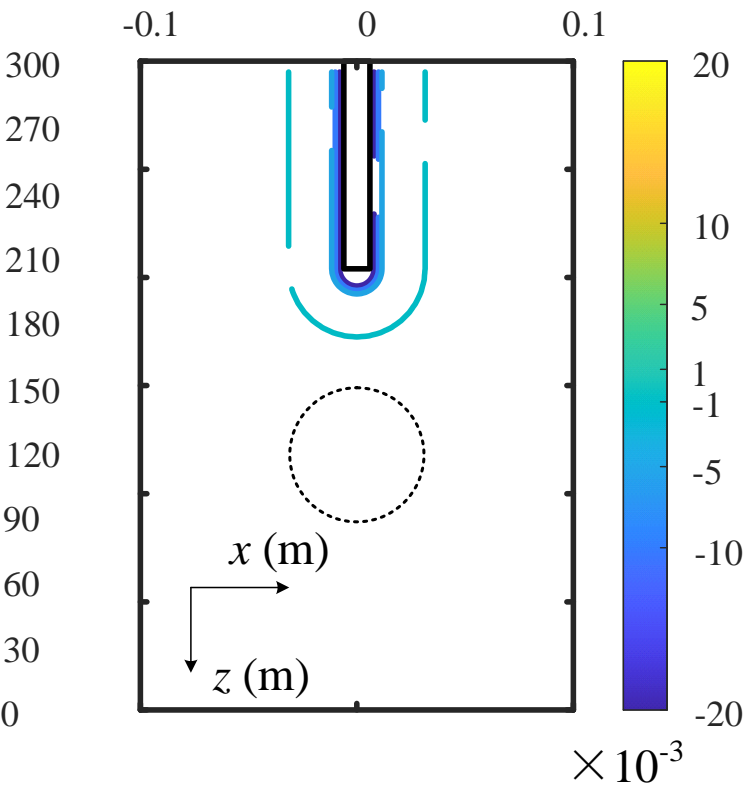

(d) Specific volume field after piling

0.1

$-0.1$

0.1

Fig. S3 Changes and cumulative stress and specific volume fields after piling

The pile end bearing capacity after installation is also estimated based on spherical cavity expansion, but the cavity radius is expanded from the pile radius to $110 \%$ of pile radius (i.e. $\left.a_{0}=6 \mathrm{~mm}, a=6.6 \mathrm{~mm}\right)$. At the position of the pile end, the initial stress condition is assumed as the average value within the plastic zone of soil around the pile tip from the pile 
installation. The calculated cavity pressure is then used to determine the pile bearing capacity following Eq. (7). For TP1-P1, the pile bearing capacity $q_{t}=5294.7 \mathrm{kPa}$. In terms of the pile load capacity, Eq. (8) is used to determine the pile tip capacity $Q_{t i p}=598.8 \mathrm{~N}$, pile shaft capacity $Q_{\text {shaft }}=780 \mathrm{~N}$ and total pile capacity $Q=1378.8 \mathrm{~N}$.

\section{S3 Tunnel volume loss}

\section{S3.1 Tunnel convergence-confinement curve}

Firstly, the initial stress and specific volume are assumed as the average value from the updated stress and specific volume fields in Fig. S3(c-d) within the range of $5 r_{t}$ from the tunnel centre, giving $p_{0, t u n}^{\prime}=151.9 \mathrm{kPa}$ and $e_{0, t u n}=0.652$.

Cylindrical cavity contraction (Yu et al., 2019) provides the tunnel convergence-confinement curve, as shown in Fig. 5. For a soil element around the cavity, the changes of mean stress and specific volume are related to the normalized displacement towards the center of the tunnel, as presented in Fig. 6.

\section{S3.2 Updated mean stress and void ratio fields}

The displacement profile from cavity contraction solution of Yu et al. (2019) is concentric to the cavity center, which is only valid for deep tunnels, whereas the tunnels in urban areas are normally buried at shallow depths with influences of ground surface. Therefore, the elastoplastic solution of Yu et al. (2019) needs to be modified to consider the surface effects. As this study takes the tunnel volume loss as a key parameter to analyze the tunnel-pile interaction, the problem is displacement-controlled, and the tunneling induced displacement field is thus vital to the analyses. To overcome the limitations on displacement fields for a shallow tunnel, the elastic solution of Strack (2002) is used to calculate a tunneling-induced displacement field. The approximate solution follows the model of Verruijt and Booker (1996), and considers both ground loss and ovalization (see Fig. S4), as well as the third part to eliminate the shear stress at the surface using the Fourier transform method. Both vertical displacement and normalized displacement $\left(u_{r} / r\right)$ contours are shown in Fig. 7. 
(a) Ground loss

(b) Ovalization

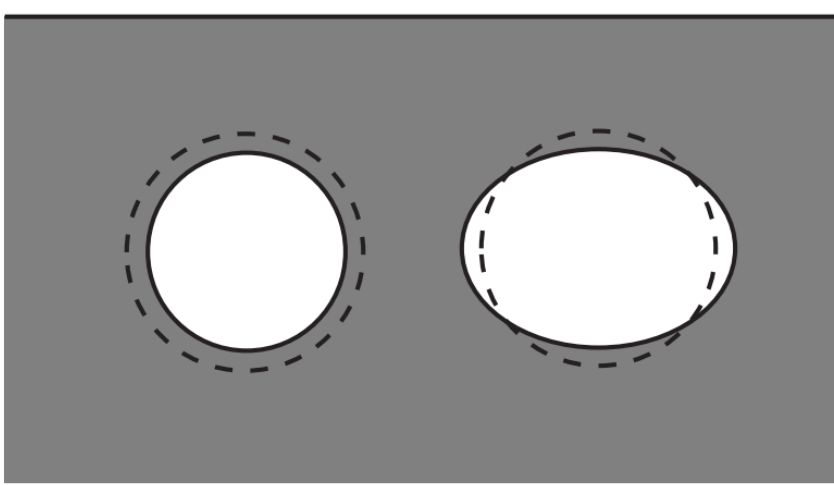

(c) Image solution for a singularity

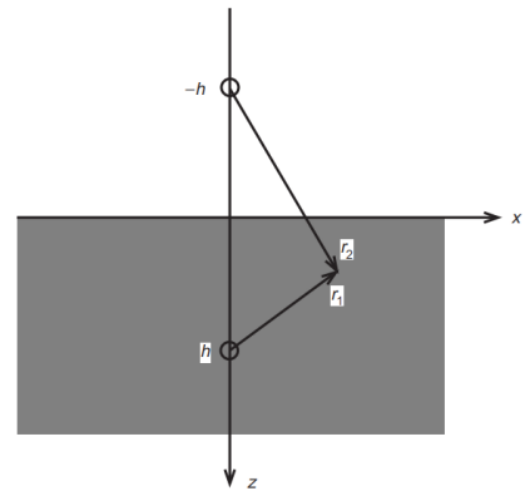

Fig. S4 Calculation model for deformation of a tunnel in an elastic half plane, following Verruijt and Booker (1996) and Strack (2002)

For each soil element in Fig. 7(b), the magnitude of normalized displacement is taken to project 96 the relevant changes $\Delta p_{c y l}^{\prime}$ and $\Delta v_{c y l}$ from the curves in Fig. 6. As the initial states vary with locations, the changes $\Delta p_{c y l}^{\prime}$ and $\Delta v_{c y l}$ are modified to yield $\Delta p^{\prime}$ and $\Delta v$ at all soil elements, following Eq. (9). Then, the contours by tunneling and by both piling and tunneling can be plotted until the ultimate volume loss $V_{l, u l t}=1.76 \%$, as shown in Fig. S5. The cumulative stress field after piling and tunneling is shown in Fig. 8(a). 
(a) Changes of stress field by tunneling

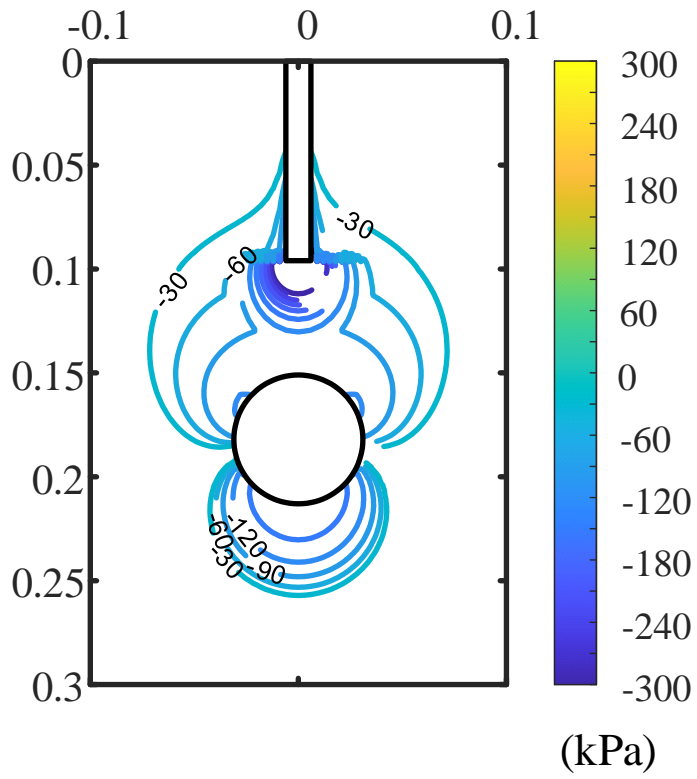

(b) Changes of $\mathrm{v}$ field by tunneling

$-0.1$

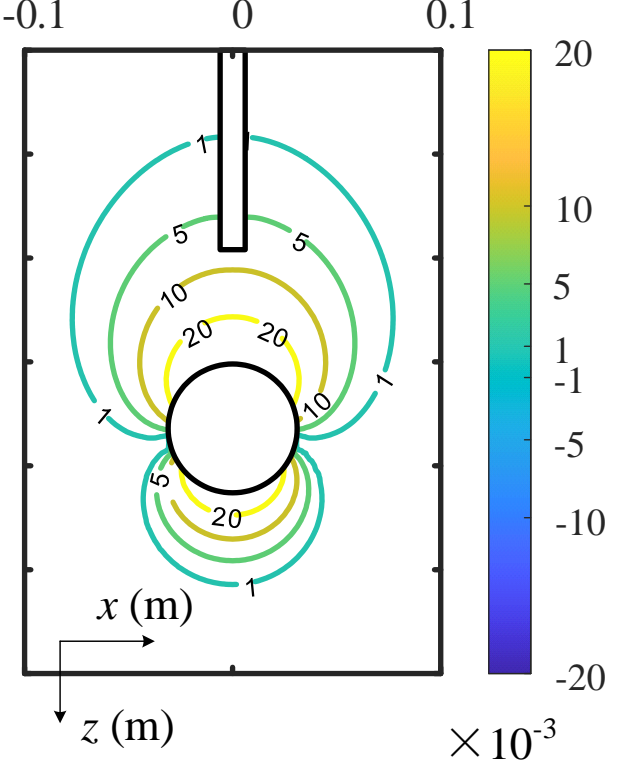

(c) Changes of stress field by piling and tunneling

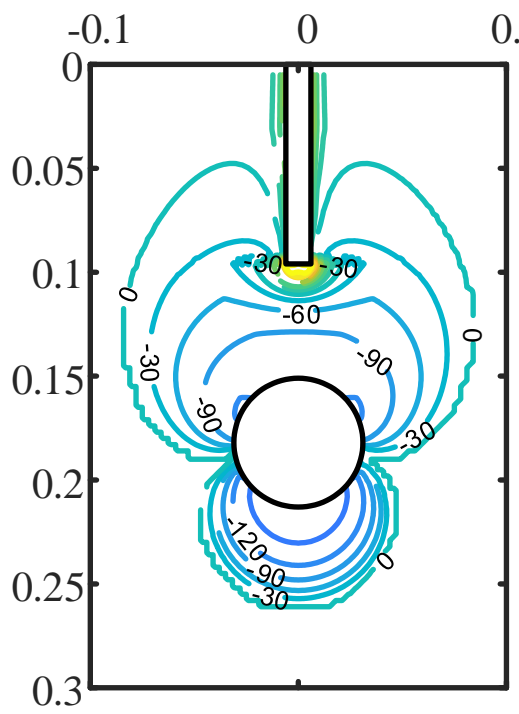

0.1

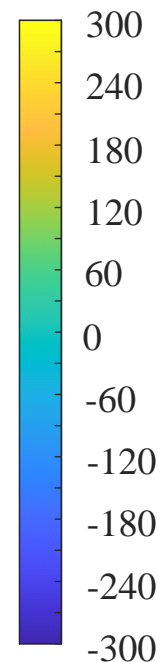

$(\mathrm{kPa})$ (d) Changes of $\mathrm{v}$ field by piling and tunneling

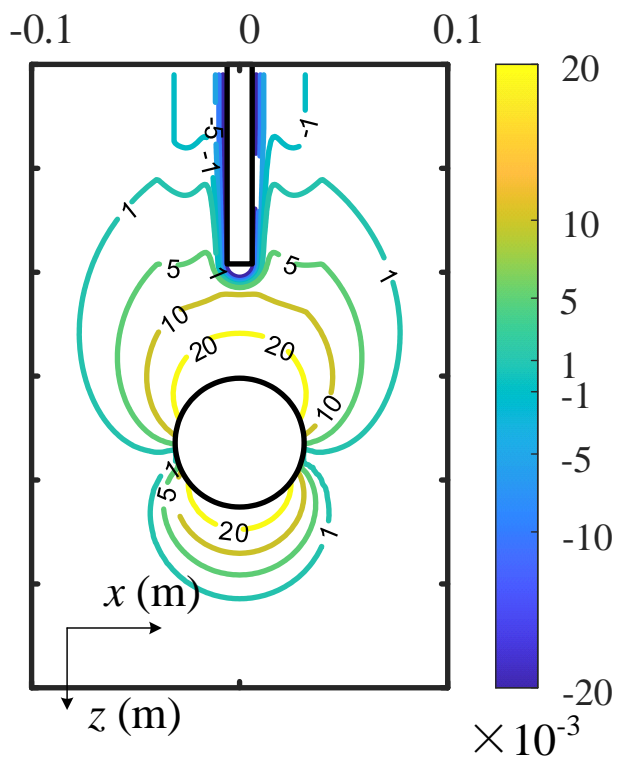

102 Fig. S5 Changes and cumulative stress and specific volume fields by: (a-b) tunneling; (c-d)

\section{S3.3 Reduced pile bearing capacity and $R_{Q}$}

105 At any tunnel volume loss, the steps in S2.4 are repeated based on the current stress and specific volume fields to estimate the mobilized pile bearing capacity. Then, the variations of $Q_{t i p}$, $Q_{\text {shaft }}$ and $Q$ with tunnel volume loss can be obtained, as presented in Fig. 9(b). The 
108 reduction factor for total capacity is then determined by $R_{Q}=Q_{V l} / Q_{0}$.

\section{S3.4 Estimation of tunneling induced settlement}

110 The tunneling induced settlement is calculated by $s_{p i l e, V l}=s_{1, V l}+s_{2, V l}$, where $s_{1, V l}$ is the

111 induced ground settlement and $s_{2, V l}$ is caused by the degradation of pile capacity. $s_{1, V l}$ can 112 be estimated from the contour of Fig. 7(a). $s_{2, V l}$ needs to evaluate the load-settlement response 113 by Eq. (10). Note that $q_{t}$ is calculated from S3.3 and $k_{i}$ is estimated based on the updated 114 stress and specific volume fields.

115 The initial safety factor $S F_{0}=1.65$ is applied to match the TP1-P1 centrifuge test, which is 116 equivalent to a service load at the pile head of $835.6 \mathrm{~N}$. As the initial shaft capacity is $117 Q_{\text {shaft }, 0}=780 \mathrm{~N}, s_{0} / b_{p}=0.0035$ based on Eq. (11). When the tunnel volume loss reaches $1181.0 \%$, for example, the pile tip stress increases to about $2500 \mathrm{kPa}\left(q_{\text {serv }, V l}=\max \left(P_{\text {load }}-\right.\right.$ $\left.119 Q_{\text {shaft }, V l}, 0\right) /\left(\pi \cdot r_{p}{ }^{2}\right)$ ) according to Eq. (12), and the normalized settlement is $s_{i} / b_{p}=$ 1200.0659 from the corresponding pile load-settlement curve (i.e. dark blue line in Fig. 9a). The 121 pile capacity degradation induced pile settlement at $V_{l, t}=1.0 \%$ is thus calculated as $s_{2, V l}=$ $122 s_{i}-s_{0} \approx 0.75 \mathrm{~mm}$. Together with the tunneling induced ground settlement at the pile tip (Fig. $1237 \mathrm{a}$ ), the total tunneling induced pile settlement is $s_{p i l e, V l}=s_{1, V l}+s_{2, V l} \approx 1.04 \mathrm{~mm}$.

125 This worked example for TP1-P1 is provided to show details on the calculations. Note that 126 some data, models, or code that support the findings of this study are available from the first author or the corresponding author upon reasonable request. 